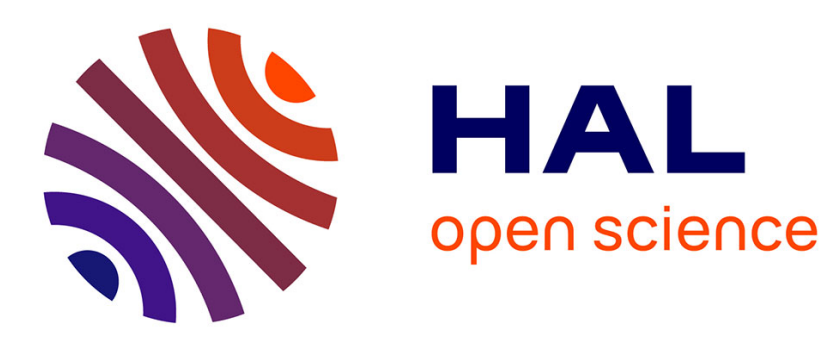

\title{
$h p$-adaptation driven by polynomial-degree-robust a posteriori error estimates for elliptic problems
}

Vít Dolejší, Alexandre Ern, Martin Vohralík

\section{To cite this version:}

Vít Dolejší, Alexandre Ern, Martin Vohralík. $h p$-adaptation driven by polynomial-degree-robust a posteriori error estimates for elliptic problems. SIAM Journal on Scientific Computing, 2016, 38 (5), pp.A3220-A3246. 10.1137/15M1026687 . hal-01165187v3

\section{HAL Id: hal-01165187 \\ https://inria.hal.science/hal-01165187v3}

Submitted on 27 Jun 2016

HAL is a multi-disciplinary open access archive for the deposit and dissemination of scientific research documents, whether they are published or not. The documents may come from teaching and research institutions in France or abroad, or from public or private research centers.
L'archive ouverte pluridisciplinaire HAL, est destinée au dépôt et à la diffusion de documents scientifiques de niveau recherche, publiés ou non, émanant des établissements d'enseignement et de recherche français ou étrangers, des laboratoires publics ou privés. 


\title{
$h p$-adaptation driven by polynomial-degree-robust a posteriori error estimates for elliptic problems*
}

\author{
Vít Dolejš̌̆ ${ }^{(1)}$, Alexandre Ern ${ }^{(2)}$, Martin Vohralík ${ }^{(3)}$ \\ (1) Charles University Prague, Faculty of Mathematics and Physics, Sokolovská 83, 18675 \\ Praha, Czech Republic, (dolejsi@karlin.mff.cuni.cz) \\ (2) Université Paris-Est, CERMICS (ENPC), 77455 Marne-la-Vallée cedex 2, France \\ (alexandre.ern@enpc.fr). \\ (3) INRIA of Paris, 2 rue Simone Iff, 75012 Paris, France (martin.vohralik@inria.fr)
}

\begin{abstract}
We devise and study experimentally adaptive strategies driven by a posteriori error estimates to select automatically both the space mesh and the polynomial degree in the numerical approximation of diffusion equations in two space dimensions. The adaptation is based on equilibrated flux estimates. These estimates are presented here for inhomogeneous Dirichlet and Neumann boundary conditions, for spatiallyvarying polynomial degree, and for mixed rectangular-triangular grids possibly containing hanging nodes. They deliver a global error upper bound with constant one and, up to data oscillation, error lower bounds on element patches with a generic constant only dependent on the mesh regularity and with a computable bound. We numerically asses the estimates and several $h p$-adaptive strategies using the interior penalty discontinuous Galerkin method. Asymptotic exactness is observed for all the symmetric, nonsymmetric (odd degrees), and incomplete variants on non-nested unstructured triangular grids for a smooth solution and uniform refinement. Exponential convergence rates are reported on nonmatching triangular grids for the incomplete version on several benchmarks with a singular solution and adaptive refinement.
\end{abstract}

Keywords: Laplace equation, a posteriori error estimate, $p$-robustness, $h p$-refinement, nonmatching grids, inhomogeneous boundary conditions, discontinuous Galerkin

\footnotetext{
*This work was partly supported by the ERC-CZ project MORE "MOdelling REvisited + MOdel REduction" LL1202. The research of V. Dolejší was supported by the Grant No. 13-00522S of the Czech Science Foundation. This author acknowledges also the membership in the Nečas Center for Mathematical Modeling ncmm.karlin.mff.cuni.cz. M. Vohralík has received funding from the European Research Council (ERC) under the European Union's Horizon 2020 research and innovation program (grant agreement No 647134 GATIPOR).
} 


\section{Introduction}

A posteriori error estimates for elliptic problems have been studied for several decades. These estimates deliver global upper bounds for the discretization error as a Hilbertian sum of local (cellwise) error indicators that are computable solely from the discrete solution. At the same time, they represent local error lower bounds, up to data oscillation, see, e.g., the recent textbook [43]. They can be devised under various forms. Among these, equilibrated flux error estimates offer the salient advantage of delivering error upper bounds with constant one. Such estimates are typically evaluated by solving local mixed finite element problems on element patches around mesh vertices, see $[6,16,21,27]$ and the references therein. Another attractive property of equilibrated flux a posteriori error estimates that was uncovered recently in the conforming finite element setting, see [6], is polynomial-degree-robustness, that is, the generic constant in the local error lower bound turns out to be uniform with respect to the polynomial degree (it only depends on the shape-regularity of the underlying meshes). This result stems from nontrivial properties of mixed finite element spaces, namely from a right inverse of the divergence operator [11, Corollary 3.4] and from a right inverse of the normal trace [12, Theorem 7.1], on triangular meshes, and from [6, Theorem 5], on rectangular meshes, whose stability properties are uniform with respect to the polynomial degree. This type of result is not expected to hold in the more popular setting of residual-based a posteriori error estimates [29]. The polynomial-degree-robustness of equilibrated flux estimates was extended recently in [21] to a unified setting encompassing many nonconforming discretizations such as nonconforming finite elements, mixed finite elements, and interior penalty discontinuous Galerkin methods. Therein, the idea is to introduce, in addition to the flux reconstruction, a conforming potential reconstruction which is also built by solving local problems in element patches around vertices.

The recent advances on equilibrated flux a posteriori error estimates have been presented in the setting of homogeneous Dirichlet boundary conditions, for uniform polynomial degree, and on matching meshes. The first contribution of this work is to extend these estimates to the practical setting of inhomogeneous Dirichlet and Neumann boundary conditions, to cover discretizations with variable polynomial degree, and to allow for mixed rectangular-triangular grids with hanging nodes. These extensions turn out to be nontrivial: possibly different polynomial degrees need to be assigned to each patch when reconstructing the flux and the potential, the local mixed finite element problems have to be suitably modified on patches touching the boundary, and the local partition of unity which combines contributions from all the vertices of a given mesh cell has to be revisited. Additionally, to treat nonmatching grids, we refine in a matching way the patches around each node which is not a hanging node, but we avoid the matching refinement of the entire grid as well as triangular subrefinements of rectangular grids, previously used in [20, Appendix] or [33]. Importantly, we still achieve global error upper bounds with constant one and polynomial-degree-robust local error lower bounds.

The second contribution of this work is to devise an $h p$-adaptive strategy driven by the above polynomial-degree-robust equilibrated flux a posteriori error estimates. Several criteria for determining whether it is preferable to perform $h$ - (mesh) or $p$ (polynomial degree) refinement have been proposed over the years, see, e.g., [1, 5, 13, $19,22,23,24,25,28,29,37,38,39,41]$, the survey in [31], and the references therein. Typically, it is natural to increase the polynomial degree where the solution is estimated to be sufficiently smooth, and to decrease the mesh size where the solution is 
estimated to be rather rough. Therefore, a key ingredient is an estimate of the local smoothness of the exact solution. In the present setting, we exploit the polynomialdegree-robustness of the estimate and combine it with three $h p$-adaptive strategies. Two of them are adaptations of techniques known from the literature [23, 41]. The third one is new and is based on the comparison of the error indicator for the current discrete solution and its local projections onto the discretization space with polynomial degree minus one and a locally coarser grid. Such an approach is well-suited to discretizations by the discontinuous Galerkin method. We assess the $h p$-adaptive strategy using the incomplete interior penalty variant on four benchmark problems with a locally singular exact solution. Our numerical results show that exponential convergence rates with respect to degrees of freedom are achieved with the three strategies, in agreement with the theoretical results from [5, 13, 28, 37, 38].

The paper is organized as follows. In Section 2, we briefly describe the setting and introduce basic notation. In Section 3, we devise and analyze equilibrated flux a posteriori error estimates for inhomogeneous Dirichlet and Neumann boundary conditions, varying polynomial degree, and nonmatching mixed rectangular-triangular grids. We devote Section 4 to a brief description of the interior penalty discontinuous Galerkin method, a discussion of some implementation aspects, and a numerical illustration of the asymptotic exactness of our a posteriori error estimators for uniform mesh and polynomial degree refinement and a smooth solution. In Section 5, we present the various $h p$-adaptive strategies. Numerical results achieving exponential convergence on several benchmark problems with singular solutions are finally presented in Section 6 .

\section{Setting}

Let $\Omega \subset \mathbb{R}^{2}$ be a polygonal domain (open, bounded, and connected set) with unit outward normal $\mathbf{n}_{\Omega}$. We suppose that $\partial \Omega$ is divided into two simply connected parts $\Gamma_{\mathrm{D}}$ and $\Gamma_{\mathrm{N}}$ with disjoint interiors, and we assume that $\left|\Gamma_{\mathrm{D}}\right|>0$. We consider the Laplace equation: find $u: \Omega \rightarrow \mathbb{R}$ such that

$$
\begin{aligned}
-\Delta u & =f & & \text { in } \Omega, \\
-\nabla u \cdot \mathbf{n}_{\Omega} & =\sigma_{\mathrm{N}} & & \text { on } \Gamma_{\mathrm{N}}, \\
u & =u_{\mathrm{D}} & & \text { on } \Gamma_{\mathrm{D}} .
\end{aligned}
$$

All what follows can be easily extended to the case where a Neumann condition is enforced on the whole boundary, by adding a zero-mean value constraint to the solution and assuming the usual compatibility condition between the data $f$ and $\sigma_{\mathrm{N}}$.

Let $H^{1}(\Omega)$ denote the Sobolev space composed of $L^{2}(\Omega)$ functions with weak gradients in $\left[L^{2}(\Omega)\right]^{2}$. Then, $H_{0, \mathrm{D}}^{1}(\Omega)$ (resp., $H_{u_{\mathrm{D}}}^{1}(\Omega)$ ) is composed of all functions in $H^{1}(\Omega)$ with zero trace (resp., with trace equal to $u_{\mathrm{D}}$ ) on $\Gamma_{\mathrm{D}}$. The variational formulation of (2.1) reads: find $u \in H_{u_{\mathrm{D}}}^{1}(\Omega)$ such that

$$
(\nabla u, \nabla v)=(f, v)-\left\langle\sigma_{\mathrm{N}}, v\right\rangle_{\Gamma_{\mathrm{N}}} \quad \forall v \in H_{0, \mathrm{D}}^{1}(\Omega)
$$

Here $(\cdot, \cdot)$ stands for the $L^{2}$-inner product on $\Omega$ and $\|\cdot\|$ for the associated norm. Similarly, $\langle\cdot, \cdot\rangle$ stands for the $L^{2}$-inner product on $\partial \Omega$. We add an index to $(\cdot, \cdot)$ and $\langle\cdot, \cdot\rangle$ for a (proper) subset of $\bar{\Omega}$. We suppose that $f \in L^{2}(\Omega), \sigma_{\mathrm{N}} \in L^{2}\left(\Gamma_{\mathrm{N}}\right)$, and $u_{\mathrm{D}} \in H^{1}\left(\Gamma_{\mathrm{D}}\right)$, where $H^{1}\left(\Gamma_{\mathrm{D}}\right)$ is the one-dimensional Sobolev space on $\Gamma_{\mathrm{D}}$.

We consider here that a given numerical discretization of (2.2) has been performed on a partition $\mathcal{T}_{h}$ of $\Omega$ such that $\cup_{K \in \mathcal{T}_{h}}=\bar{\Omega}$ and where each element $K \in \mathcal{T}_{h}$ is either a 
closed triangle or a closed rectangle. We suppose that the intersection of the interiors of two distinct elements is empty, but we allow for nonmatching meshes, i.e., the intersection of two different mesh elements can be a node of only one of them or a part of an edge of some of them. This gives rise to so-called hanging nodes which are not vertices of all elements by which they are shared, see Figure 1.

The edges of the mesh $\mathcal{T}_{h}$ form the set $\mathcal{E}_{h}$, with $\mathcal{E}_{h}^{\text {ext }}$ the edges lying on the boundary of $\Omega$. We suppose that the interior of each boundary edge lies entirely either in $\Gamma_{\mathrm{D}}$ or $\Gamma_{\mathrm{N}}$ and denote the corresponding subsets of $\mathcal{E}_{h}^{\text {ext }}$ by $\mathcal{E}_{h}^{\text {ext,D }}$ and $\mathcal{E}_{h}^{\text {ext, } \mathrm{N}}$, respectively. Similarly, $\mathcal{V}_{h}$ stands for all non-hanging vertices of the mesh $\mathcal{T}_{h}$ and $\mathcal{V}_{h}^{\text {ext,D }}\left(\mathcal{V}_{h}^{\text {ext, N }}\right.$ respectively) for the vertices which lie on some Dirichlet (Neumann) boundary edge. Note that $\mathcal{V}_{h}^{\text {ext,D }} \cap \mathcal{V}_{h}^{\text {ext, } \mathrm{N}}$ is not empty unless $\Gamma_{\mathrm{D}}=\partial \Omega$; vertices on the interface between $\Gamma_{\mathrm{D}}$ and $\Gamma_{\mathrm{N}}$ lie both in $\mathcal{V}_{h}^{\text {ext,D }}$ and $\mathcal{V}_{h}^{\text {ext,N }}$ in our notation. We will consider the subset $\mathcal{V}_{h}^{\text {ext,D }}$ for the flux reconstruction and the subset $\mathcal{V}_{h}^{\text {ext,N }}$ for the potential reconstruction. Let $\mathcal{V}_{e}$ stand for the two vertices of the edge $e \in \mathcal{E}_{h}$. Finally, all edges of an element $K \in \mathcal{T}_{h}$ are denoted by $\mathcal{E}_{K}$ and those edges that lie in $\Gamma_{\mathrm{D}}\left(\Gamma_{\mathrm{N}}\right)$ by $\mathcal{E}_{K}^{\mathrm{D}}$ $\left(\mathcal{E}_{K}^{\mathrm{N}}\right)$. The jump operator $\llbracket \cdot \rrbracket$ yields the difference of the traces of the argument from the two mesh elements that share $e \in \mathcal{E}_{h}^{\text {int }}$ (evaluated along a fixed unit normal $\mathbf{n}_{e}$ of $e$ ) and the actual trace on $e \in \mathcal{E}_{h}^{\text {ext }}$. Similarly, the average operator $\{\cdot\}$ yields the mean value of the traces from adjacent mesh elements on inner edges and the actual trace on boundary edges.

Let $\mathrm{R}_{\frac{\pi}{2}}:=\left(\begin{array}{cc}0 & -1 \\ 1 & 0\end{array}\right)$ be the matrix of rotation by $\frac{\pi}{2}$. We use the convention $\mathrm{R}_{\frac{\pi}{2}} \mathbf{n}_{\Omega}=\mathbf{t}_{\Omega}$ to link the unit exterior normal and tangential vectors, and similarly on subdomains of $\Omega$. We let

$$
H^{1}\left(\mathcal{T}_{h}\right):=\left\{v \in L^{2}(\Omega) ;\left.v\right|_{K} \in H^{1}(K) \forall K \in \mathcal{T}_{h}\right\}
$$

be the broken Sobolev space with respect to the (nonmatching) mesh $\mathcal{T}_{h}$ and denote the broken (elementwise) weak gradient by $\nabla$. Similarly, $\nabla \cdot$ stands for the broken weak divergence and $\mathrm{R}_{\frac{\pi}{2}} \nabla$ for the broken weak curl, $\left.\left(\mathrm{R}_{\frac{\pi}{2}} \nabla v\right)\right|_{K}=\left.\left(-\partial_{y} v, \partial_{x} v\right)^{t}\right|_{K}$ for $v \in H^{1}\left(\mathcal{T}_{h}\right)$ and all $K \in \mathcal{T}_{h}$. We will also work with piecewise polynomials: if $K$ is a triangle, we let $\mathbb{R}_{p}(K):=\mathbb{P}_{p}(K)$ be the space of polynomials of total degree at most $p \geq 0$. If $K$ is a rectangle, we let $\mathbb{R}_{p}(K):=\mathbb{Q}_{p}(K)$ be the space of polynomials of degree at most $p$ in each variable; $\mathbb{Q}_{p, p^{\prime}}(K), p, p^{\prime} \geq 0$, specifies the degrees separately. Finally, for a finite-dimensional subspace $V_{h}$ of $L^{2}(\Omega)$, we denote by $\Pi_{V_{h}}$ the $L^{2}(\Omega)$ orthogonal projection onto $V_{h}$, and similarly on various subsets of $\Omega$. We will also denote by $\Pi_{e}^{0}$ the $L^{2}(e)$-orthogonal projection onto constants on a given edge $e \in \mathcal{E}_{h}$.

We make the following assumption covering a large variety of practical meshes:

Assumption 2.1 (Nonmatching meshes). For each non-hanging node a $\in \mathcal{V}_{h}$, we suppose that there exists a unique "hat" function $\psi_{\mathbf{a}}$ which is such that it is globally continuous, it belongs to $\mathbb{R}_{1}(K)$ on each element $K \in \mathcal{T}_{h}$, and takes the value 1 at the vertex $\mathbf{a}$ and the value 0 at all other non-hanging vertices. The values at the hanging nodes are then obtained by taking the value from the element of which $\mathbf{a}$ is not a vertex. We denote by $\omega_{\mathbf{a}}$ the support of $\psi_{\mathbf{a}}$ and by $\mathcal{T}_{\mathbf{a}}$ the set of elements in $\overline{\omega_{\mathbf{a}}}$ that we call patch. We crucially suppose that

$$
\left.\sum_{\mathbf{a} \in \mathcal{V}_{K}} \psi_{\mathbf{a}}\right|_{K}=\left.1\right|_{K} \quad \forall K \in \mathcal{T}_{h},
$$

where $\mathcal{V}_{K}$ stands for the set of all non-hanging nodes $\mathbf{a} \in \mathcal{V}_{h}$ of the original mesh $\mathcal{T}_{h}$ such that the element $K$ lies in the patch $\mathcal{T}_{\mathbf{a}}$. For matching meshes, these are simply all 


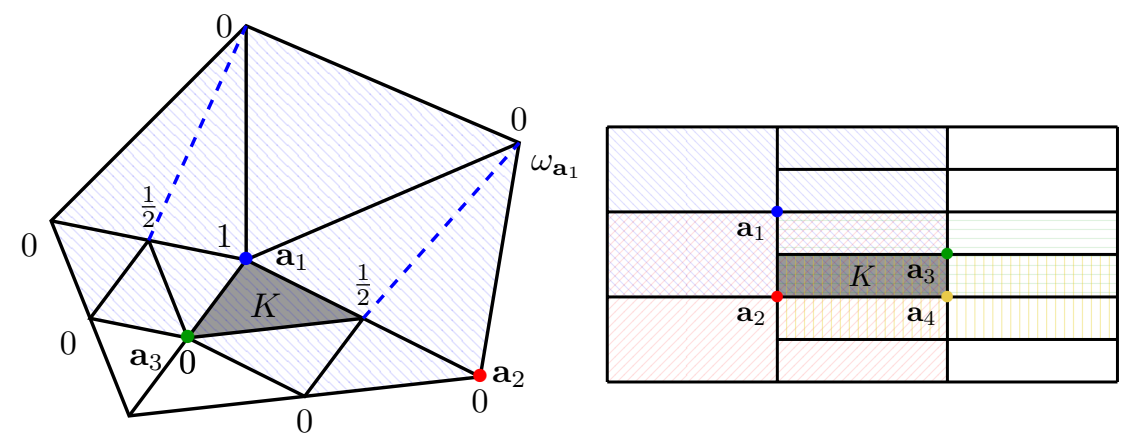

Fig. 1: Examples of nonmatching triangular (left) and rectangular (right) meshes. For the element $K$ in grey, we mark by $\bullet$ the non-hanging nodes $\mathbf{a}_{i}$ from $\mathcal{V}_{K}$. The patch $\omega_{\mathbf{a}_{1}}$ with the values of the hat function $\psi_{\mathbf{a}_{1}}$ and the matching submesh $\widehat{\mathcal{T}}_{\mathbf{a}}$ (left) and all the patches $\omega_{\mathbf{a}_{i}}$ (right)

the vertices of the element $K$, and $\mathcal{T}_{\mathbf{a}}$ is the usual patch of all mesh elements sharing the node $\mathbf{a} \in \mathcal{V}_{h}$. Finally, we let $\widehat{\mathcal{T}}_{\mathbf{a}}$ be a matching submesh of $\mathcal{T}_{\mathbf{a}}$, where triangles can only be refined into triangles and rectangles into rectangles. We suppose that $\widehat{\mathcal{T}}_{\mathbf{a}}$ is uniformly shape-regular, i.e., there exists a constant $\kappa_{\hat{\mathcal{T}}}$ such that the ratio $\hat{h}_{\hat{K}} / \varrho_{\hat{K}}$ is uniformly bounded by $\kappa_{\widehat{\mathcal{T}}}$ for all elements $\hat{K} \in \widehat{\mathcal{T}}_{\mathbf{a}}$ and for all non-hanging nodes $\mathbf{a} \in \mathcal{V}_{h}$. Here $\hat{h}_{\hat{K}}$ is the diameter of $\hat{K}$ and $\varrho_{\hat{K}}$ the diameter of the largest ball inscribed in $\hat{K}$. Note that the union of $\widehat{\mathcal{T}}_{\mathbf{a}}$ over all non-hanging nodes $\mathbf{a} \in \mathcal{V}_{h}$ does not need to form a matching refinement of $\mathcal{T}_{h}$, see Figure 1.

\section{Equilibrated flux a posteriori error estimates for inhomogeneous boundary conditions, varying polynomial degree, and nonmatching meshes}

We present in this section a posteriori error estimates of equilibrated flux type which extend previous results to inhomogeneous boundary conditions, varying polynomial degree, and nonmatching mixed rectangular-triangular meshes.

\subsection{Potential and flux reconstructions}

Let $u_{h} \in H^{1}\left(\mathcal{T}_{h}\right)$ be the approximate solution. Let $\mathfrak{G}\left(u_{h}\right) \in\left[L^{2}(\Omega)\right]^{2}$ represent its gradient: typically, either $\mathfrak{G}\left(u_{h}\right)$ is given by the broken weak gradient $\nabla u_{h}$ or by a discrete gradient also taking into account the jumps in $u_{h}$, see (3.26) below for an example. In practice, $u_{h}$ and $\mathfrak{G}\left(u_{h}\right)$ are piecewise polynomials, see assumption (3.28) below. They come from a specific numerical method, cf. Section 4.1. In general, $u_{h} \notin H^{1}(\Omega)$ and $-\mathfrak{G}\left(u_{h}\right) \notin \mathbf{H}(\operatorname{div}, \Omega)$, the space of $\left[L^{2}(\Omega)\right]^{2}$ functions with weak divergence in $L^{2}(\Omega)$. This leads us to:

Definition 3.1 (Potential reconstruction). We call a potential reconstruction any 
function $s_{\hat{h}}$ constructed from $u_{h}$ which satisfies

$$
\begin{aligned}
& s_{\hat{h}} \in H^{1}(\Omega) \cap C^{0}(\bar{\Omega}), \\
& s_{\hat{h}}(\mathbf{a})=u_{\mathrm{D}}(\mathbf{a}) \quad \forall \mathbf{a} \in \mathcal{V}_{h}^{\text {ext,D }} .
\end{aligned}
$$

Definition 3.2 (Equilibrated flux reconstruction). We call an equilibrated flux reconstruction any function $\boldsymbol{\sigma}_{\hat{h}}$ constructed from $\mathfrak{G}\left(u_{h}\right)$ which satisfies

$$
\begin{array}{ll}
\boldsymbol{\sigma}_{\hat{h}} \in \mathbf{H}(\operatorname{div}, \Omega), & \\
\left(\nabla \cdot \boldsymbol{\sigma}_{\hat{h}}, 1\right)_{K}=(f, 1)_{K} \quad \forall K \in \mathcal{T}_{h}, \\
\left\langle\boldsymbol{\sigma}_{\hat{h}} \cdot \mathbf{n}_{\Omega}, 1\right\rangle_{e}=\left\langle\sigma_{\mathrm{N}}, 1\right\rangle_{e} \quad \forall e \in \mathcal{E}_{h}^{\text {ext,N }} .
\end{array}
$$

The continuity of $s_{\hat{h}}$ imposed in (3.1a) is needed in (3.1b) to take point values; we also notice that (3.2c) requires that $\left\langle\boldsymbol{\sigma}_{\hat{h}} \cdot \mathbf{n}_{\Omega}, 1\right\rangle_{e}$ be meaningful. In practice, $s_{\hat{h}}$ and $\boldsymbol{\sigma}_{\hat{h}}$ are piecewise polynomials, so that these requirements are readily met. Notice that we employ the subscript $\hat{h}$ to indicate that $s_{\hat{h}}$ and $\boldsymbol{\sigma}_{\hat{h}}$ are constructed using the refined matching patches $\widehat{\mathcal{T}}_{\mathbf{a}}$ from Assumption 2.1 .

\subsection{A general a posteriori error estimate}

Our first important result is the generalization of [21, Theorem 3.3] to problem (2.1), cf. also [2, 3, 9, 26, 34, 35] and the references therein. Recall that if $K$ is a triangle or a rectangle from the mesh $\mathcal{T}_{h}$ and $e$ one of its edges, the following trace inequality holds:

$$
\left\|v-\Pi_{e}^{0} v\right\|_{e} \leq C_{\mathrm{t}, K, e} h_{e}^{1 / 2}\|\nabla v\|_{K} \quad \forall v \in H^{1}(K) .
$$

It is shown in [32, Lemma 3.5] that $C_{\mathrm{t}, K, e}^{2}=C_{\mathrm{t}} h_{K}^{2} /|K|$, where $C_{\mathrm{t}} \approx 0.77708$ if $K$ is a triangle and $C_{\mathrm{t}} \approx 0.31950$ if $K$ is a rectangle.

Theorem 3.3 (General a posteriori error estimate). Let $u$ be the weak solution of (2.2). Let $u_{h} \in H^{1}\left(\mathcal{T}_{h}\right)$ be an arbitrary approximation of $u$, with $\mathfrak{G}\left(u_{h}\right) \in\left[L^{2}(\Omega)\right]^{2}$ an approximation of $\nabla u$. Let $s_{\hat{h}}$ be a potential reconstruction in the sense of Definition 3.1 and $\boldsymbol{\sigma}_{\hat{h}}$ an equilibrated flux reconstruction in the sense of Definition 3.2. Then,

$$
\left\|\nabla u-\mathfrak{G}\left(u_{h}\right)\right\|^{2} \leq \eta^{2}:=\sum_{K \in \mathcal{T}_{h}} \eta_{K}^{2}
$$

with

$$
\begin{aligned}
& \eta_{K}^{2}:=(\underbrace{\left\|\mathfrak{G}\left(u_{h}\right)+\boldsymbol{\sigma}_{\hat{h}}\right\|_{K}}_{\eta_{\mathrm{CR}, K}, \text { constitutive rel. }}+\underbrace{\frac{h_{K}}{\pi}\left\|f-\nabla \cdot \boldsymbol{\sigma}_{\hat{h}}\right\|_{K}}_{\eta_{\mathrm{osc}, K} \text {, data osc. }}+\underbrace{\sum_{e \in \mathcal{E}_{K}^{\mathrm{N}}} C_{\mathrm{t}, K, e} h_{e}^{1 / 2}\left\|\boldsymbol{\sigma}_{\hat{h}} \cdot \mathbf{n}_{\Omega}-\sigma_{\mathrm{N}}\right\|_{e}}_{\eta_{\Gamma_{\mathrm{N}}, K}, \text { Neumann } \mathrm{BC}})^{2} \\
& +(\underbrace{\left\|\mathfrak{G}\left(u_{h}\right)-\nabla s_{\hat{h}}\right\|_{K}}_{\eta_{\mathrm{NC}, K}, \text { pot. nonconformity }}+\underbrace{\left.\min _{v \in H^{1}(K), \begin{array}{c}
\left.v\right|_{\partial K \cap \Gamma_{\mathrm{D}}}=u_{\mathrm{D}}-s_{\hat{h}} \\
\left.v\right|_{\partial K \backslash \Gamma_{\mathrm{D}}}=0
\end{array}}\|\nabla v\|_{K}\right)^{2}}_{\eta_{\Gamma_{\mathrm{D}}, K}, \text { Dirichlet BC }} .
\end{aligned}
$$

Proof. Let $\nabla s$ be the projection of $\mathfrak{G}\left(u_{h}\right)$ into $\nabla H_{u_{\mathrm{D}}}^{1}(\Omega)$, i.e., $s \in H_{u_{\mathrm{D}}}^{1}(\Omega)$ and $(\nabla s, \nabla v)=\left(\mathfrak{G}\left(u_{h}\right), \nabla v\right)$ for all $v \in H_{0, \mathrm{D}}^{1}(\Omega)$. This leads to the Pythagorean equality

$$
\left\|\nabla u-\mathfrak{G}\left(u_{h}\right)\right\|^{2}=\|\nabla(u-s)\|^{2}+\left\|\nabla s-\mathfrak{G}\left(u_{h}\right)\right\|^{2} .
$$


The first term in (3.5) can be rewritten as follows, since $(u-s) \in H_{0, \mathrm{D}}^{1}(\Omega)$ :

$$
\begin{aligned}
\|\nabla(u-s)\| & =\sup _{\varphi \in H_{0, \mathrm{D}}^{1}(\Omega) ;\|\nabla \varphi\|=1}(\nabla(u-s), \nabla \varphi), \\
& =\sup _{\varphi \in H_{0, \mathrm{D}}^{1}(\Omega) ;\|\nabla \varphi\|=1}\left(\nabla u-\mathfrak{G}\left(u_{h}\right), \nabla \varphi\right), \\
& =\sup _{\varphi \in H_{0, \mathrm{D}}^{1}(\Omega) ;\|\nabla \varphi\|=1}\left\{(f, \varphi)-\left\langle\sigma_{\mathrm{N}}, \varphi\right\rangle_{\Gamma_{\mathrm{N}}}-\left(\mathfrak{G}\left(u_{h}\right), \nabla \varphi\right)\right\},
\end{aligned}
$$

where we have used the definition of $s$ and (2.2). Fix $\varphi \in H_{0, \mathrm{D}}^{1}(\Omega)$ with $\|\nabla \varphi\|=1$. Adding and subtracting $\left(\boldsymbol{\sigma}_{\hat{h}}, \nabla \varphi\right)$, where $\boldsymbol{\sigma}_{\hat{h}}$ is the equilibrated flux reconstruction in the sense of Definition 3.2, and using the Green theorem, we infer that

$(f, \varphi)-\left\langle\sigma_{\mathrm{N}}, \varphi\right\rangle_{\Gamma_{\mathrm{N}}}-\left(\mathfrak{G}\left(u_{h}\right), \nabla \varphi\right)=\left(f-\nabla \cdot \boldsymbol{\sigma}_{\hat{h}}, \varphi\right)+\left\langle\boldsymbol{\sigma}_{\hat{h}} \cdot \mathbf{n}_{\Omega}-\sigma_{\mathrm{N}}, \varphi\right\rangle_{\Gamma_{\mathrm{N}}}-\left(\mathfrak{G}\left(u_{h}\right)+\boldsymbol{\sigma}_{\hat{h}}, \nabla \varphi\right)$.

The first and last terms above are treated exactly as in the proof of [21, Theorem 3.3], using in particular the equilibration (3.2b). For the middle term, we observe that

$$
\begin{aligned}
\left\langle\boldsymbol{\sigma}_{\hat{h}} \cdot \mathbf{n}_{\Omega}-\sigma_{\mathrm{N}}, \varphi\right\rangle_{\Gamma_{\mathrm{N}}} & =\sum_{K \in \mathcal{T}_{h}} \sum_{e \in \mathcal{E}_{K}^{\mathrm{N}}}\left\langle\boldsymbol{\sigma}_{\hat{h}} \cdot \mathbf{n}_{\Omega}-\sigma_{\mathrm{N}}, \varphi\right\rangle_{e}=\sum_{K \in \mathcal{T}_{h}} \sum_{e \in \mathcal{E}_{K}^{\mathrm{N}}}\left\langle\boldsymbol{\sigma}_{\hat{h}} \cdot \mathbf{n}_{\Omega}-\sigma_{\mathrm{N}}, \varphi-\Pi_{e}^{0} \varphi\right\rangle_{e} \\
& \leq \sum_{K \in \mathcal{T}_{h}} \sum_{e \in \mathcal{E}_{K}^{\mathrm{N}}}\left\{\left\|\boldsymbol{\sigma}_{\hat{h}} \cdot \mathbf{n}_{\Omega}-\sigma_{\mathrm{N}}\right\|_{e} C_{\mathrm{t}, K, e} h_{e}^{1 / 2}\|\nabla \varphi\|_{K}\right\},
\end{aligned}
$$

owing to (3.2c), the Cauchy-Schwarz inequality, and (3.3). Note that only those elements having (at least) one edge located on the Neumann boundary are concerned. This leads to the terms composing the first line of (3.4b).

Consider now the second term in (3.5). Proceeding as in [26, Section 4.1], we infer that

$$
\begin{aligned}
& \left\|\nabla s-\mathfrak{G}\left(u_{h}\right)\right\|^{2}=\min _{w \in H_{u_{\mathrm{D}}}^{1}(\Omega)}\left\|\nabla w-\mathfrak{G}\left(u_{h}\right)\right\|^{2} \\
& \leq \min _{w \in H_{u_{\mathrm{D}}}^{1}}(\Omega) \sum_{K \in \mathcal{T}_{h}}\left(\left\|\nabla s_{\hat{h}}-\mathfrak{G}\left(u_{h}\right)\right\|_{K}+\left\|\nabla\left(w-s_{\hat{h}}\right)\right\|_{K}\right)^{2} \\
& \leq \sum_{K \in \mathcal{T}_{h}}\left(\left\|\nabla s_{\hat{h}}-\mathfrak{G}\left(u_{h}\right)\right\|_{K}+\min _{\substack{\left.\left.w\right|_{\partial K \cap \Gamma_{\mathrm{D}}=u_{\mathrm{D}}} \\
w\right|_{\partial K \backslash \Gamma_{\mathrm{D}}=s_{\hat{h}}}}}\left\|\nabla\left(w-s_{\hat{h}}\right)\right\|_{K}\right)^{2},
\end{aligned}
$$

where the first inequality follows by localization on mesh elements and the triangle inequality, and the second one by restricting the global minimum to elementwise minima over functions $w \in H^{1}(K)$ with values on $\partial K$ fixed respectively to $u_{\mathrm{D}}$ or $s_{\hat{h}}$, thanks to conditions (3.1a) and (3.1b). Note that the elements concerned by the minimization are those having (at least) one edge located on the Dirichlet boundary. This leads to the terms composing the second line of (3.4b).

The expression for the general Dirichlet boundary condition error from Theorem 3.3 is not computable. We now derive a computable upper bound for this quantity. The proof is skipped since it follows that in [9, Theorem 5.1]; it consists in bounding the minimum by considering a function given by the Dirichlet boundary misfit on the concerned edges and extending it linearly to zero at the cell barycenter using polar coordinates. 


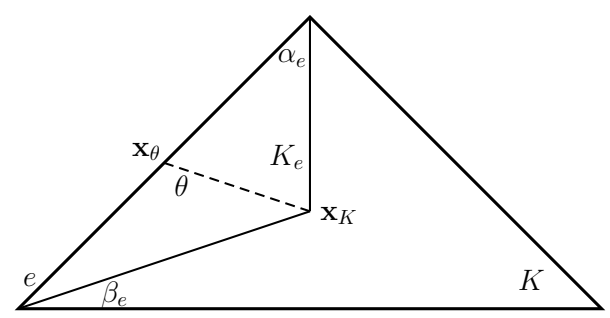

Fig. 2: Notation for the inhomogeneous Dirichlet boundary condition estimate, triangle example

Theorem 3.4 (Inhomogeneous Dirichlet boundary condition estimate). Let $K \in \mathcal{T}_{h}$ be such that $\left|\partial K \cap \Gamma_{\mathrm{D}}\right|>0$. Let $\mathbf{x}_{K}$ denote the barycenter of $K$. For each $e \in \mathcal{E}_{K}^{\mathrm{D}}$, consider the polar coordinates $r, \theta$ centered at $\mathbf{x}_{K}$, where the triangle $K_{e}$ given by the edge $e$ and the point $\mathbf{x}_{K}$ is described by $\theta \in\left[\alpha_{e}, \beta_{e}\right]$ and $r \in\left[0, R_{e}(\theta)\right] ; R_{e}(\theta)$ is thus the distance between $\mathbf{x}_{K}$ and $\mathbf{x}_{\theta} \in e$, see Figure 2 for $K$ being a triangle. Set $g_{e}(\theta):=\left(u_{\mathrm{D}}-s_{\hat{h}}\right)\left(\mathbf{x}_{\theta}\right)$ and denote by' the differentiation with respect to $\theta$. Then

$$
\begin{aligned}
& \min _{v \in H^{1}(K),} \min _{\substack{\left.\partial K \cap \Gamma_{\mathrm{D}} \\
v\right|_{\partial K \backslash \Gamma_{\mathrm{D}}}=u_{\mathrm{D}}-s_{\hat{h}}}}\|\nabla v\|_{K} \\
& \leq \sum_{e \in \mathcal{E}_{K}^{\mathrm{D}}}\left\{\frac{1}{2} \int_{\alpha_{e}}^{\beta_{e}}\left\{\left[g_{e}(\theta)\right]^{2}+\left[\left(g_{e}^{\prime}(\theta) R_{e}(\theta)-g_{e}(\theta) R_{e}^{\prime}(\theta)\right) / R_{e}(\theta)\right]^{2}\right\} \mathrm{d} \theta\right\}^{1 / 2} .
\end{aligned}
$$

This computable estimate is of higher order whenever $u_{\mathrm{D}}$ has enough regularity, see the discussions in $[26,9]$.

\subsection{Reconstructions from local problems with variable polynomial degree on matching patches}

In this section, we extend the material of [21, Section 3.1.3] in two ways. First, we treat meshes with hanging nodes. Our concept does not require the existence of a global matching refinement of the original mesh $\mathcal{T}_{h}$ and is rather different from the previous approaches in [20, Appendix], [2,3], and [33], see also the references therein. In particular, the present potential and flux reconstructions are constructed in the individually refined matching patches $\widehat{\mathcal{T}}_{\mathbf{a}}$ around each non-hanging node a $\in \mathcal{V}_{h}$, see Assumption 2.1. Second, the reconstructions on each patch $\widehat{\mathcal{T}}_{\mathbf{a}}$ can be assigned a specific polynomial degree $p_{\mathbf{a}} \geq 0$ (see (3.30) below). Then, we let $\mathbf{V}_{\hat{h}}\left(\omega_{\mathbf{a}}\right)$ be the Raviart-Thomas mixed finite element space of degree $p_{\mathbf{a}}$ on the mesh $\widehat{\mathcal{T}}_{\mathbf{a}}$ of $\omega_{\mathbf{a}}$. Functions in this space belong to $\left[\mathbb{P}_{p_{\mathbf{a}}}(\hat{K})\right]^{2}+\mathbb{P}_{p_{\mathbf{a}}}(\hat{K}) \mathbf{x}$ if $\hat{K}$ is a triangle and to $\mathbb{Q}_{p_{\mathbf{a}}+1, p_{\mathbf{a}}}(\hat{K}) \times \mathbb{Q}_{p_{\mathbf{a}}, p_{\mathbf{a}}+1}(\hat{K})$ if $\hat{K}$ is a rectangle in $\widehat{\mathcal{T}}_{\mathbf{a}}$. Moreover, their normal trace over the edges inside $\widehat{\mathcal{T}}_{\mathbf{a}}$ is in $\mathbb{P}_{p_{\mathbf{a}}}(\hat{e})$ and is continuous $[8,36]$. Recalling the notation of Section 2 , let $Q_{\hat{h}}\left(\omega_{\mathbf{a}}\right)$ be the space of discontinuous piecewise polynomials on $\widehat{\mathcal{T}}_{\mathbf{a}}$ being in $\mathbb{R}_{p_{\mathbf{a}}}(\hat{K})$ on each $\hat{K} \in \widehat{\mathcal{T}}_{\mathbf{a}}$. Alternatively, Brezzi-Douglas-Marini spaces could also be considered, see [21, Remark 3.21].

The reconstruction of an equilibrated flux $\boldsymbol{\sigma}_{\hat{h}}$ according to Definition 3.2, for varying polynomial degree, general boundary conditions, and nonmatching mixed meshes, 
takes the following form:

Definition 3.5 (Flux reconstruction $\left.\boldsymbol{\sigma}_{\hat{h}}\right)$. Assume that $\mathfrak{G}\left(u_{h}\right)$ satisfies the hat-function orthogonality on the patches $\omega_{\mathbf{a}}$ around non-haging nodes of Assumption 2.1:

$$
\left(\mathfrak{G}\left(u_{h}\right), \nabla \psi_{\mathbf{a}}\right)_{\omega_{\mathbf{a}}}=\left(f, \psi_{\mathbf{a}}\right)_{\omega_{\mathbf{a}}}-\left\langle\sigma_{\mathrm{N}}, \psi_{\mathbf{a}}\right\rangle_{\Gamma_{\mathrm{N}}} \quad \forall \mathbf{a} \in \mathcal{V}_{h} \backslash \mathcal{V}_{h}^{\text {ext, }, \mathrm{D}}
$$

For each $\mathbf{a} \in \mathcal{V}_{h}$, prescribe $\boldsymbol{\varsigma}_{\hat{h}}^{\mathbf{a}} \in \mathbf{V}_{\hat{h}, \mathrm{~N}}^{\mathbf{a}}$ and $\bar{r}_{\hat{h}}^{\mathbf{a}} \in Q_{\hat{h}}^{\mathbf{a}}$ by solving

$$
\begin{aligned}
\left(\boldsymbol{\varsigma}_{\hat{h}}^{\mathbf{a}}, \mathbf{v}_{\hat{h}}\right)_{\omega_{\mathbf{a}}}-\left(\bar{r}_{\hat{h}}^{\mathbf{a}}, \nabla \cdot \mathbf{v}_{\hat{h}}\right)_{\omega_{\mathbf{a}}} & =-\left(\psi_{\mathbf{a}} \mathfrak{G}\left(u_{h}\right), \mathbf{v}_{\hat{h}}\right)_{\omega_{\mathbf{a}}} & & \forall \mathbf{v}_{\hat{h}} \in \mathbf{V}_{\hat{h}}^{\mathbf{a}}, \\
\left(\nabla \cdot \boldsymbol{\varsigma}_{\hat{h}}^{\mathbf{a}}, q_{\hat{h}}\right)_{\omega_{\mathbf{a}}} & =\left(\psi_{\mathbf{a}} f-\nabla \psi_{\mathbf{a}} \cdot \mathfrak{G}\left(u_{h}\right), q_{\hat{h}}\right)_{\omega_{\mathbf{a}}} & & \forall q_{\hat{h}} \in Q_{\hat{h}}^{\mathbf{a}}
\end{aligned}
$$

with the following spaces: for all $\mathbf{a} \in \mathcal{V}_{h}^{\text {int }}$,

$$
\begin{aligned}
\mathbf{V}_{\hat{h}, \mathrm{~N}}^{\mathbf{a}} & :=\mathbf{V}_{\hat{h}}^{\mathbf{a}}:=\left\{\mathbf{v}_{\hat{h}} \in \mathbf{V}_{\hat{h}}\left(\omega_{\mathbf{a}}\right) ;\left.\mathbf{v}_{\hat{h}} \cdot \mathbf{n}_{\omega_{\mathbf{a}}}\right|_{\partial \omega_{\mathbf{a}}}=0\right\}, \\
Q_{\hat{h}}^{\mathbf{a}} & :=\left\{q_{\hat{h}} \in Q_{\hat{h}}\left(\omega_{\mathbf{a}}\right) ;\left(q_{\hat{h}}, 1\right)_{\omega_{\mathbf{a}}}=0\right\},
\end{aligned}
$$

and, for all $\mathbf{a} \in \mathcal{V}_{h}^{\text {ext }}$, with $\tilde{g}_{\mathrm{N}}^{\mathbf{a}}:=\Pi_{\mathbf{V}_{\hat{h}}\left(\omega_{\mathbf{a}}\right) \cdot \mathbf{n}}\left(\psi_{\mathbf{a}} \sigma_{\mathrm{N}}\right)$,

$$
\begin{aligned}
\mathbf{V}_{\hat{h}, \mathrm{~N}}^{\mathbf{a}} & :=\left\{\mathbf{v}_{\hat{h}} \in \mathbf{V}_{\hat{h}}\left(\omega_{\mathbf{a}}\right) ;\left.\mathbf{v}_{\hat{h}} \cdot \mathbf{n}_{\omega_{\mathbf{a}}}\right|_{\partial \omega_{\mathbf{a}} \backslash \partial \Omega}=0, \mathbf{v}_{\hat{h}} \cdot \mathbf{n}_{\omega_{\mathbf{a}}} \mid \partial \omega_{\mathbf{a}} \cap \Gamma_{\mathrm{N}}=\tilde{g}_{\mathrm{N}}^{\mathbf{a}}\right\}, \\
\mathbf{V}_{\hat{h}}^{\mathbf{a}} & :=\left\{\mathbf{v}_{\hat{h}} \in \mathbf{V}_{\hat{h}}\left(\omega_{\mathbf{a}}\right) ;\left.\mathbf{v}_{\hat{h}} \cdot \mathbf{n}_{\omega_{\mathbf{a}}}\right|_{\partial \omega_{\mathbf{a}} \backslash \partial \Omega}=0, \mathbf{v}_{\hat{h}} \cdot \mathbf{n}_{\omega_{\mathbf{a}}} \mid \partial \omega_{\mathbf{a}} \cap \Gamma_{\mathrm{N}}=0\right\},
\end{aligned}
$$

while

$$
\begin{array}{ll}
Q_{\hat{h}}^{\mathbf{a}}:=\left\{q_{\hat{h}} \in Q_{\hat{h}}\left(\omega_{\mathbf{a}}\right) ;\left(q_{\hat{h}}, 1\right)_{\omega_{\mathbf{a}}}=0\right\} & \mathbf{a} \in \mathcal{V}_{h}^{\text {ext }} \backslash \mathcal{V}_{h}^{\text {ext,D }}, \\
Q_{\hat{h}}^{\mathbf{a}}:=Q_{\hat{h}}\left(\omega_{\mathbf{a}}\right) & \mathbf{a} \in \mathcal{V}_{h}^{\text {ext,D }} .
\end{array}
$$

Then, extending $\boldsymbol{\varsigma}_{\hat{h}}^{\mathbf{a}}$ by zero outside $\omega_{\mathbf{a}}$, set

$$
\boldsymbol{\sigma}_{\hat{h}}:=\sum_{\mathbf{a} \in \mathcal{V}_{h}} \boldsymbol{\varsigma}_{\hat{h}}^{\mathbf{a}}
$$

The above local problems only differ from those of [6, equation (9)] or [21, Construction 3.4] in two ways. First, whereas the datum $\mathfrak{G}\left(u_{h}\right)$ and the hat function $\psi_{\mathbf{a}}$ are linked with the original (nonmatching) mesh $\mathcal{T}_{h}$, the equilibration is being performed on the refined matching patches $\widehat{\mathcal{T}}_{\mathbf{a}}$ around each non-hanging node $\mathbf{a} \in \mathcal{V}_{h}$. Note that $\widehat{\mathcal{T}}_{\mathbf{a}}$ typically has more elements than just those sharing the (non-hanging) vertex $\mathbf{a}$. The second difference appears for vertices which lie on the Neumann boundary $\Gamma_{\mathrm{N}}$, i.e., whenever a lies on $\partial \Omega$ and $\left|\partial \omega_{\mathbf{a}} \cap \Gamma_{\mathrm{N}}\right|>0$ : an inhomogeneous Neumann boundary condition is encoded in the space $\mathbf{V}_{\hat{h}, \mathbf{N}}^{\mathbf{a}}$ of $(3.9 \mathrm{a})$ whereby $\left.\boldsymbol{\varsigma}_{\hat{h}}^{\mathbf{a}} \cdot \mathbf{n}_{\omega_{\mathbf{a}}}\right|_{\partial \omega_{\mathbf{a}} \cap \Gamma_{\mathbf{N}}}$ is enforced to be the polynomial projection of $\psi_{\mathbf{a}} \sigma_{\mathrm{N}}$ onto the space of normal traces of the local mixed finite element space $\mathbf{V}_{\hat{h}}\left(\omega_{\mathbf{a}}\right)$. Problem (3.7) for $\mathbf{a} \in \mathcal{V}_{h}^{\text {ext }}$ is a pure Neumann problem when $\partial \omega_{\mathbf{a}} \cap \Gamma_{\mathrm{N}}=\partial \omega_{\mathbf{a}} \cap \partial \Omega$, i.e., when the whole boundary of $\omega_{\mathbf{a}}$ lying on $\partial \Omega$ is on the Neumann boundary. This happens if and only if $\mathbf{a} \in \mathcal{V}_{h}^{\text {ext }} \backslash \mathcal{V}_{h}^{\text {ext,D }}$ in our notation. The Neumann compatibility condition then requests that

$$
\left(\psi_{\mathbf{a}} f-\nabla \psi_{\mathbf{a}} \cdot \mathfrak{G}\left(u_{h}\right), 1\right)_{\omega_{\mathbf{a}}}=\left\langle\Pi_{\mathbf{V}_{\hat{h}}\left(\omega_{\mathbf{a}}\right) \cdot \mathbf{n}}\left(\psi_{\mathbf{a}} \sigma_{\mathrm{N}}\right), 1\right\rangle_{\partial \omega_{\mathbf{a}} \cap \Gamma_{\mathbf{N}}} .
$$

Noting that $\left\langle\Pi_{\mathbf{V}_{\hat{h}}\left(\omega_{\mathbf{a}}\right) \cdot \mathbf{n}}\left(\psi_{\mathbf{a}} \sigma_{\mathrm{N}}\right), 1\right\rangle_{\partial \omega_{\mathbf{a}} \cap \Gamma_{\mathrm{N}}}=\left\langle\psi_{\mathbf{a}} \sigma_{\mathrm{N}}, 1\right\rangle_{\Gamma_{\mathrm{N}}}$, this is nothing but (3.6) for $\mathbf{a} \in \mathcal{V}_{h}^{\text {ext }} \backslash \mathcal{V}_{h}^{\text {ext,D }}$. Shall $\left|\partial \omega_{\mathbf{a}} \cap \Gamma_{\mathrm{D}}\right|>0$ for a boundary vertex, we have a local Neumann-Dirichlet problem, with the normal trace of $\boldsymbol{\varsigma}_{\hat{h}}^{\mathbf{a}}$ not prescribed on $\partial \omega_{\mathbf{a}} \cap \Gamma_{\mathrm{D}}$. 
Lemma 3.6 (Properties of $\boldsymbol{\sigma}_{\hat{h}}$ ). The following holds:

$$
\begin{array}{ll}
\boldsymbol{\sigma}_{\hat{h}} \in \mathbf{H}(\operatorname{div}, \Omega), & \\
\left(f-\nabla \cdot \boldsymbol{\sigma}_{\hat{h}}, v_{h}\right)_{K}=0 & \forall K \in \mathcal{T}_{h}, \forall v_{h} \in \mathbb{R}_{\min _{\mathbf{a} \in \mathcal{V}_{K}} p_{\mathbf{a}}}(K), \\
\left\langle\boldsymbol{\sigma}_{\hat{h}} \cdot \mathbf{n}_{\Omega}-\sigma_{\mathrm{N}}, v_{h}\right\rangle_{e}=0 & \forall e \in \mathcal{E}_{h}^{\mathrm{ext}, \mathrm{N}}, \forall v_{h} \in \mathbb{P}_{\min _{\mathbf{a} \in \mathcal{V}_{e}} p_{\mathbf{a}}(e) .}
\end{array}
$$

Thus, in particular the three properties in (3.2) are satisfied.

Proof. The fluxes $\boldsymbol{\varsigma}_{\hat{h}}^{\mathbf{a}} \in \mathbf{V}_{\hat{h}, \mathrm{~N}}^{\mathbf{a}}$ have a zero normal trace over $\partial \omega_{\mathbf{a}} \backslash \partial \Omega$, so that their extension by zero outside $\omega_{\mathbf{a}}$ is in $\mathbf{H}(\operatorname{div}, \Omega)$, even though each refined matching patch $\widehat{\mathcal{T}}_{\mathbf{a}}$ is defined independently from the rest of the domain. Next, the Neumann compatibility condition (3.6) implies that (3.7b) actually holds for all functions in $Q_{\hat{h}}\left(\omega_{\mathbf{a}}\right)$. Now fix an element $K$ in the original (nonmatching) partition $\mathcal{T}_{h}$ and a polynomial

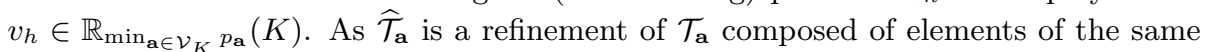
(triangular/rectangular) type owing to Assumption 2.1, the function $v_{h}$ is in the space $Q_{\hat{h}}\left(\omega_{\mathbf{a}}\right)$ for all the refined matching patches $\widehat{\mathcal{T}}_{\mathbf{a}}$ such that $\mathbf{a} \in \mathcal{V}_{K}$. Employing that $\left.\boldsymbol{\sigma}_{\hat{h}}\right|_{K}=\left.\sum_{\mathbf{a} \in \mathcal{V}_{K}} \boldsymbol{\varsigma}_{\hat{h}}^{\mathbf{a}}\right|_{K}$ and $(3.7 \mathrm{~b})$, we find

$$
\left(f-\nabla \cdot \boldsymbol{\sigma}_{\hat{h}}, v_{h}\right)_{K}=\sum_{\mathbf{a} \in \mathcal{V}_{K}}\left(\psi_{\mathbf{a}} f-\nabla \cdot \boldsymbol{\varsigma}_{\hat{h}}^{\mathbf{a}}, v_{h}\right)_{K}=\sum_{\mathbf{a} \in \mathcal{V}_{K}}\left(\nabla \psi_{\mathbf{a}} \cdot \mathfrak{G}\left(u_{h}\right), v_{h}\right)_{K}=0,
$$

where one crucially uses the partition of unity (2.3). Finally, let $e \in \mathcal{E}_{h}^{\text {ext,N }}$ be a Neumann edge and let $v_{h} \in \mathbb{P}_{\min _{\mathbf{a} \in \mathcal{V}_{e}} p_{\mathbf{a}}}(e)$. Employing that $\left.\boldsymbol{\sigma}_{\hat{h}}\right|_{e}=\sum_{\mathbf{a} \in \mathcal{V}_{e}} \boldsymbol{\varsigma}_{\hat{h}}^{\mathbf{a}}$ and using the normal trace condition imposed on $\mathbf{V}_{\hat{h}, \mathrm{~N}}^{\mathbf{a}}$ in $(3.9 \mathrm{a})$, we infer that $\left\langle\boldsymbol{\sigma}_{\hat{h}} \cdot \mathbf{n}_{\Omega}, v_{h}\right\rangle_{e}=$ $\sum_{\mathbf{a} \in \mathcal{V}_{e}}\left\langle\boldsymbol{\varsigma}_{\hat{h}}^{\mathbf{a}} \cdot \mathbf{n}_{\Omega}, v_{h}\right\rangle_{e}=\sum_{\mathbf{a} \in \mathcal{V}_{e}}\left\langle\psi_{\mathbf{a}} \sigma_{\mathrm{N}}, v_{h}\right\rangle_{e}=\left\langle\sigma_{\mathrm{N}}, v_{h}\right\rangle_{e}$

Remark 3.7 (Local flux minimization). Similarly to [21, Remark 3.7], Definition 3.5 can be equivalently stated as:

$$
\boldsymbol{\varsigma}_{\hat{h}}^{\mathbf{a}}:=\arg \min _{\mathbf{v}_{\hat{h}} \in \mathbf{V}_{\hat{h}, \mathbf{N}}^{\mathbf{a}}, \nabla \cdot \mathbf{v}_{\hat{h}}=\Pi_{Q_{\hat{h}}}^{\mathbf{a}}\left(\psi_{\mathbf{a}} f-\nabla \psi_{\mathbf{a}} \cdot \mathfrak{G}\left(u_{h}\right)\right)}\left\|\psi_{\mathbf{a}} \mathfrak{G}\left(u_{h}\right)+\mathbf{v}_{\hat{h}}\right\|_{\omega_{\mathbf{a}}} \quad \forall \mathbf{a} \in \mathcal{V}_{h}
$$

We now turn to the potential reconstruction. Being in two space dimensions, we can construct $s_{\hat{h}}$ via the same local problems (3.7), upon merely replacing the righthand sides and adjusting the spaces $\mathbf{V}_{\hat{h}}^{\mathbf{a}}$ and $Q_{\hat{h}}^{\mathbf{a}}$ close to the boundary. This turns out to be equivalent to a primal conforming finite element solve, see Remark 3.10 below.

Definition 3.8 (Potential reconstruction $s_{\hat{h}}$ ). For each $\mathbf{a} \in \mathcal{V}_{h}$, prescribe $\boldsymbol{\varsigma}_{\hat{h}}^{\mathbf{a}} \in \mathbf{V}_{\hat{h}, \mathrm{~N}}^{\mathbf{a}}$ and $\bar{r}_{\hat{h}}^{\mathbf{a}} \in Q_{\hat{h}}^{\mathbf{a}}$ by solving

$$
\begin{array}{rlrl}
\left(\boldsymbol{\varsigma}_{\hat{h}}^{\mathbf{a}}, \mathbf{v}_{\hat{h}}\right) \omega_{\mathbf{a}}- & \left(\bar{r}_{\hat{h}}^{\mathbf{a}}, \nabla \cdot \mathbf{v}_{\hat{h}}\right)_{\omega_{\mathbf{a}}}=-\left(\mathrm{R}_{\frac{\pi}{2}} \nabla\left(\psi_{\mathbf{a}} u_{h}\right), \mathbf{v}_{\hat{h}}\right) \omega_{\mathbf{a}} & & \forall \mathbf{v}_{\hat{h}} \in \mathbf{V}_{\hat{h}}^{\mathbf{a}}, \\
\left(\nabla \cdot \boldsymbol{\varsigma}_{\hat{h}}^{\mathbf{a}}, q_{\hat{h}}\right)_{\omega_{\mathbf{a}}}=0 & & \forall q_{\hat{h}} \in Q_{\hat{h}}^{\mathbf{a}}
\end{array}
$$

with the following spaces: for all $\mathbf{a} \in \mathcal{V}_{h}^{\text {int }}$,

$$
\begin{aligned}
\mathbf{V}_{\hat{h}, \mathrm{~N}}^{\mathbf{a}} & :=\mathbf{V}_{\hat{h}}^{\mathbf{a}}:=\left\{\mathbf{v}_{\hat{h}} \in \mathbf{V}_{\hat{h}}\left(\omega_{\mathbf{a}}\right) ;\left.\mathbf{v}_{\hat{h}} \cdot \mathbf{n}_{\omega_{\mathbf{a}}}\right|_{\partial \omega_{\mathbf{a}}}=0\right\}, \\
Q_{\hat{h}}^{\mathbf{a}} & :=\left\{q_{\hat{h}} \in Q_{\hat{h}}\left(\omega_{\mathbf{a}}\right) ;\left(q_{\hat{h}}, 1\right)_{\omega_{\mathbf{a}}}=0\right\},
\end{aligned}
$$

and, for all $\mathbf{a} \in \mathcal{V}_{h}^{\text {ext }}$, with $\tilde{g}_{\mathrm{D}}^{\mathbf{a}}:=\Pi_{\mathbf{V}_{\hat{h}}\left(\omega_{\mathbf{a}}\right) \cdot \mathbf{n}}\left(\nabla\left(\psi_{\mathbf{a}} u_{\mathrm{D}}\right) \cdot \mathbf{t}_{\Omega}\right)$,

$$
\begin{aligned}
\mathbf{V}_{\hat{h}, \mathrm{~N}}^{\mathbf{a}} & :=\left\{\mathbf{v}_{\hat{h}} \in \mathbf{V}_{\hat{h}}\left(\omega_{\mathbf{a}}\right) ;\left.\mathbf{v}_{\hat{h}} \cdot \mathbf{n}_{\omega_{\mathbf{a}}}\right|_{\partial \omega_{\mathbf{a}} \backslash \partial \Omega}=0,\left.\mathbf{v}_{\hat{h}} \cdot \mathbf{n}_{\omega_{\mathbf{a}}}\right|_{\partial \omega_{\mathbf{a}} \cap \Gamma_{\mathrm{D}}}=\tilde{g}_{\mathrm{D}}^{\mathbf{a}}\right\}, \\
\mathbf{V}_{\hat{h}}^{\mathbf{a}} & :=\left\{\mathbf{v}_{\hat{h}} \in \mathbf{V}_{\hat{h}}\left(\omega_{\mathbf{a}}\right) ;\left.\mathbf{v}_{\hat{h}} \cdot \mathbf{n}_{\omega_{\mathbf{a}}}\right|_{\partial \omega_{\mathbf{a}} \backslash \partial \Omega}=0,\left.\mathbf{v}_{\hat{h}} \cdot \mathbf{n}_{\omega_{\mathbf{a}}}\right|_{\partial \omega_{\mathbf{a}} \cap \Gamma_{\mathrm{D}}}=0\right\},
\end{aligned}
$$


while

$$
\begin{array}{ll}
Q_{\hat{h}}^{\mathbf{a}}:=\left\{q_{\hat{h}} \in Q_{\hat{h}}\left(\omega_{\mathbf{a}}\right) ;\left(q_{\hat{h}}, 1\right)_{\omega_{\mathbf{a}}}=0\right\} & \mathbf{a} \in \mathcal{V}_{h}^{\text {ext }} \backslash \mathcal{V}_{h}^{\text {ext }, \mathrm{N}}, \\
Q_{\hat{h}}^{\mathbf{a}}:=Q_{\hat{h}}\left(\omega_{\mathbf{a}}\right) & \mathbf{a} \in \mathcal{V}_{h}^{\text {ext, } \mathrm{N}}
\end{array}
$$

Then, there is a continuous, piecewise polynomial function $s_{\hat{h}}^{\mathbf{a}}$ on $\omega_{\mathbf{a}}$ such that

$$
\begin{aligned}
&-\mathrm{R}_{\frac{\pi}{2}} \nabla s_{\hat{h}}^{\mathbf{a}}:=\boldsymbol{\varsigma}_{\hat{h}}^{\mathbf{a}}, \\
&\left.s_{\hat{h}}^{\mathbf{a}}\right|_{\partial \omega_{\mathbf{a}} \backslash \partial \Omega}:=0,
\end{aligned}
$$

and extending $s_{\hat{h}}^{\mathbf{a}}$ by zero outside $\omega_{\mathbf{a}}$, we set $s_{\hat{h}}:=\sum_{\mathbf{a} \in \mathcal{V}_{h}} s_{\hat{h}}^{\mathbf{a}}$.

Note that for boundary vertices $\mathbf{a} \in \mathcal{V}_{h}^{\text {ext }} \backslash \mathcal{V}_{h}^{\text {ext,N }}$ (which are such that $\partial \omega_{\mathbf{a}} \cap \Gamma_{\mathrm{D}}=$ $\partial \omega_{\mathbf{a}} \cap \partial \Omega$, i.e., the whole boundary of $\omega_{\mathbf{a}}$ lying on $\partial \Omega$ is on the Dirichlet boundary), (3.14) is a pure Neumann problem: the normal trace of $\boldsymbol{\varsigma}_{\hat{h}}^{\mathbf{a}}$ on $\partial \omega_{\mathbf{a}} \cap \Gamma_{\mathrm{D}}$ is prescribed by the (polynomial projection of the) tangential trace of $\nabla\left(\psi_{\mathbf{a}} u_{\mathrm{D}}\right)$. The Neumann compatibility condition then requests that

$$
\left\langle\tilde{g}_{\mathrm{D}}^{\mathbf{a}}, 1\right\rangle_{\partial \omega_{\mathbf{a}} \cap \Gamma_{\mathrm{D}}}=\left\langle\Pi_{\mathbf{V}_{\hat{h}}\left(\omega_{\mathbf{a}}\right) \cdot \mathbf{n}}\left(\nabla\left(\psi_{\mathbf{a}} u_{\mathrm{D}}\right) \cdot \mathbf{t}_{\Omega}\right), 1\right\rangle_{\partial \omega_{\mathbf{a}} \cap \Gamma_{\mathrm{D}}}=0 .
$$

This is immediate developing the above right-hand side since

$$
\begin{aligned}
\left\langle\tilde{g}_{\mathrm{D}}^{\mathrm{a}}, 1\right\rangle_{\partial \omega_{\mathbf{a}} \cap \Gamma_{\mathrm{D}}} & =-\left\langle\mathrm{R}_{\frac{\pi}{2}} \nabla\left(\psi_{\mathbf{a}} u_{\mathrm{D}}\right) \cdot \mathbf{n}_{\Omega}, 1\right\rangle_{\partial \omega_{\mathbf{a}} \cap \Gamma_{\mathrm{D}}}=-\left\langle\mathrm{R}_{\frac{\pi}{2}} \nabla\left(\psi_{\mathbf{a}} u_{\mathrm{D}}\right) \cdot \mathbf{n}_{\omega_{\mathbf{a}}}, 1\right\rangle_{\partial \omega_{\mathbf{a}}} \\
& =-\left(\nabla \cdot\left(\mathrm{R}_{\frac{\pi}{2}} \nabla\left(\psi_{\mathbf{a}} u_{\mathrm{D}}\right)\right), 1\right)_{\omega_{\mathbf{a}}}-\left(\mathrm{R}_{\frac{\pi}{2}} \nabla\left(\psi_{\mathbf{a}} u_{\mathrm{D}}\right), \nabla 1\right)_{\omega_{\mathbf{a}}}=0,
\end{aligned}
$$

for any smooth enough extension $u_{\mathrm{D}}$ of the Dirichlet boundary condition $u_{\mathrm{D}}$. Shall $\left|\partial \omega_{\mathbf{a}} \cap \Gamma_{\mathrm{N}}\right|>0,(3.14)$ is a local Neumann-Dirichlet problem, with the normal trace of $\boldsymbol{\varsigma}_{\hat{h}}^{\mathbf{a}}$ not prescribed on $\partial \omega_{\mathbf{a}} \cap \Gamma_{\mathrm{N}}$.

Lemma 3.9 (Properties of $s_{\hat{h}}$ ). Conditions (3.1a) and (3.1b) are satisfied. Moreover, the following holds for all $e \in \mathcal{E}_{h}^{\text {ext,D }}$ :

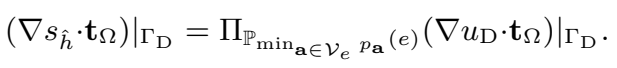

Proof. Condition (3.1a) is met by construction, since all $s_{\hat{h}}^{\mathbf{a}}$ are continuous and zero on the part of the boundary of $\omega_{\mathbf{a}}$ lying inside $\Omega$. We next show (3.21). Let $e \in \mathcal{E}_{h}^{\text {ext,D }}$

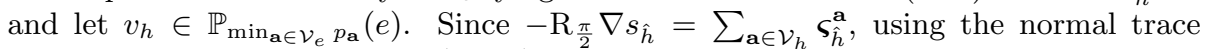
condition imposed on $\mathbf{V}_{\hat{h}, \mathrm{~N}}^{\mathbf{a}}$ in (3.16a), we infer that

$$
\left\langle-\mathrm{R}_{\frac{\pi}{2}} \nabla s_{\hat{h}} \cdot \mathbf{n}_{\Omega}, v_{h}\right\rangle_{e}=\sum_{\mathbf{a} \in \mathcal{V}_{e}}\left\langle\boldsymbol{s}_{\hat{h}}^{\mathbf{a}} \cdot \mathbf{n}_{\Omega}, v_{h}\right\rangle_{e}=\sum_{\mathbf{a} \in \mathcal{V}_{e}}\left\langle\nabla\left(\psi_{\mathbf{a}} u_{\mathrm{D}}\right) \cdot \mathbf{t}_{\Omega}, v_{h}\right\rangle_{e}=\left\langle\nabla u_{\mathrm{D}} \cdot \mathbf{t}_{\Omega}, v_{h}\right\rangle_{e}
$$

and (3.21) follows from $-\mathrm{R}_{\frac{\pi}{2}} \nabla s_{\hat{h}} \cdot \mathbf{n}_{\Omega}=\nabla s_{\hat{h}} \cdot \mathbf{t}_{\Omega}$. To show (3.1b), we reason as follows: for each $\mathbf{a} \in \mathcal{V}_{h}^{\text {ext,D }}, \nabla s_{\hat{h}}^{\mathbf{a}} \cdot \mathbf{t}_{\Omega}$ preserves edgewise mean values of $\nabla\left(\psi_{\mathbf{a}} u_{\mathrm{D}}\right) \cdot \mathbf{t}_{\Omega}$ for all $e \in \mathcal{E}_{h}^{\text {ext,D }}$ contained in $\partial \omega_{\mathbf{a}}$. This follows as above from (3.18a) and (3.16a). Moreover, by $(3.18 \mathrm{~b}), s_{\hat{h}}^{\mathbf{a}}\left(\mathbf{a}^{\prime}\right)=0=\left(\psi_{\mathbf{a}} u_{\mathrm{D}}\right)\left(\mathbf{a}^{\prime}\right)$ for the other vertices $\mathbf{a}^{\prime}$ of $\omega_{\mathbf{a}}$ lying on $\partial \omega_{\mathbf{a}} \cap \Gamma_{\mathrm{D}}$. Thus, $s_{\hat{h}}^{\mathbf{a}}(\mathbf{a})=\left(\psi_{\mathbf{a}} u_{\mathrm{D}}\right)(\mathbf{a})=u_{\mathrm{D}}(\mathbf{a})$, and the conclusion follows from the definition of $s_{\hat{h}}$. 
Remark 3.10 (Local potential minimization). As in [21, Remark 3.10], Definition 3.8 can be equivalently stated as: $\boldsymbol{\varsigma}_{\hat{h}}^{\mathbf{a}}:=\arg \min _{\mathbf{v}_{\hat{h}} \in \mathbf{V}_{\hat{h}, \mathrm{~N}}^{\mathbf{a}}, \nabla \cdot \mathbf{v}_{\hat{h}}=0}\left\|\mathrm{R}_{\frac{\pi}{2}} \nabla\left(\psi_{\mathbf{a}} u_{h}\right)+\mathbf{v}_{\hat{h}}\right\|_{\omega_{\mathbf{a}}}$ for all $\mathbf{a} \in \mathcal{V}_{h}$. Then, a discrete primal formulation is

$$
s_{\hat{h}}^{\mathbf{a}}:=\arg \min _{v_{h} \in V_{h, \mathrm{D}}^{\mathbf{a}}}\left\|\nabla\left(\psi_{\mathbf{a}} u_{h}-v_{h}\right)\right\|_{\omega_{\mathbf{a}}} \quad \forall \mathbf{a} \in \mathcal{V}_{h},
$$

where $V_{h, \mathrm{D}}^{\mathbf{a}}$ denotes continuous piecewise polynomials on $\widehat{\mathcal{T}}_{\mathbf{a}}$ such that $\mathrm{R}_{\frac{\pi}{2}} \nabla V_{h, \mathrm{D}}^{\mathbf{a}}=$ $\mathbf{V}_{\hat{h}, \mathrm{~N}}^{\mathbf{a}}$. Functions in $V_{h, \mathrm{D}}^{\mathbf{a}}$ are polynomials of total degree $p_{\mathbf{a}}+1$ on each triangle from $\widehat{\mathcal{T}}_{\mathbf{a}}$ and of degree at most $p_{\mathbf{a}}+1$ in each variable on each rectangle from $\widehat{\mathcal{T}}_{\mathbf{a}}$. They are zero on the whole boundary $\partial \omega_{\mathbf{a}}$ for $\mathbf{a} \in \mathcal{V}_{h}^{\text {int }}$, zero on $\partial \omega_{\mathbf{a}} \backslash \partial \Omega$ for $\mathbf{a} \in \mathcal{V}_{h}^{\text {ext }} \backslash \mathcal{V}_{h}^{\text {ext,D }}$, and, for $\mathbf{a} \in \mathcal{V}_{h}^{\text {ext,D }}$, the Dirichlet boundary condition on $\partial \omega_{\mathbf{a}} \backslash \Gamma_{\mathrm{N}}$ is given by

$$
\begin{aligned}
\left.\left(\nabla v_{h} \cdot \mathbf{t}_{\Omega}\right)\right|_{e} & =\left.\Pi_{\mathbf{V}_{\hat{h}}\left(\omega_{\mathbf{a}}\right) \cdot \mathbf{n}_{\omega_{\mathbf{a}}}}\left(\nabla\left(\psi_{\mathbf{a}} u_{\mathrm{D}}\right) \cdot \mathbf{t}_{\Omega}\right)\right|_{e} \quad \forall e \in \mathcal{E}_{h}^{\mathrm{ext}, \mathrm{D}}, e \subset \partial \omega_{\mathbf{a}}, \\
v_{h}(\mathbf{a}) & =u_{\mathrm{D}}(\mathbf{a}),\left.\quad v_{h}\right|_{\partial \omega_{\mathbf{a}} \backslash \partial \Omega}=0 .
\end{aligned}
$$

Let $V_{h}^{\mathbf{a}}$ be as $V_{h, \mathrm{D}}^{\mathbf{a}}$, with a homogeneous Dirichlet boundary condition everywhere on $\partial \omega_{\mathbf{a}} \backslash \Gamma_{\mathrm{N}}$. Then, (3.22) is further equivalent to finding $s_{\hat{h}}^{\mathbf{a}} \in V_{h, \mathrm{D}}^{\mathbf{a}}$ such that

$$
\left(\nabla s_{\hat{h}}^{\mathbf{a}}, \nabla v_{h}\right)=\left(\nabla\left(\psi_{\mathbf{a}} u_{h}\right), \nabla v_{h}\right) \quad \forall v_{h} \in V_{h}^{\mathbf{a}} .
$$

Remark 3.11 (Reconstruction on a matching refinement of $\mathcal{T}_{h}$ ). When $\cup_{\mathbf{a} \in \mathcal{V}_{h}} \widehat{\mathcal{T}}_{\mathbf{a}}$ is a matching refinement of $\mathcal{T}_{h}$ (this often happens on triangular meshes but typically not on rectangular meshes), it is possible to use reconstructions on the patches $\omega_{\hat{\mathbf{a}}}$ of all vertices sharing the given refinement node $\hat{\mathbf{a}}$ instead of the larger patches $\omega_{\mathbf{a}}$. For the potential, there is indeed no problem, as the source term and Neumann data in (3.14) are both homogeneous, so that the Neumann compatibility holds. For the flux, we set $\boldsymbol{\sigma}_{\hat{h}}:=\boldsymbol{\sigma}_{1, \hat{h}}+\boldsymbol{\sigma}_{2, \hat{h}}, \boldsymbol{\sigma}_{1, \hat{h}}:=\sum_{\mathbf{a} \in \mathcal{V}_{h}} \boldsymbol{\varsigma}_{1, \hat{h}}^{\mathbf{a}}$ and $\boldsymbol{\sigma}_{2, \hat{h}}:=\sum_{\mathbf{a} \in \mathcal{V}_{h}} \boldsymbol{\varsigma}_{2, \hat{h}}^{\mathbf{a}}$ from the proof of Theorem 3.12 below, for which Lemma 3.6 still holds so that we can easily evaluate $\eta_{\mathrm{osc}, K}$ and $\eta_{\Gamma_{\mathrm{N}}, K}$ in $(3.4 \mathrm{~b})$, whereas we estimate $\left\|\mathfrak{G}\left(u_{h}\right)+\boldsymbol{\sigma}_{\hat{h}}\right\| \leq$ $\left\|\mathfrak{G}\left(u_{h}\right)+\boldsymbol{\sigma}_{1, \hat{h}}\right\|+\left\|\boldsymbol{\sigma}_{2, \hat{h}}\right\|$ and bound $\left\|\boldsymbol{\sigma}_{2, \hat{h}}\right\|$ as in (3.39) to avoid its actual construction.

\subsection{Local efficiency}

In this section, we extend the polynomial-degree-robust, local error lower bound from [21, Theorem 3.17] to inhomogeneous boundary conditions, variable polynomial degree, and nonmatching mixed meshes under Assumption 2.1. This last extension turns out to be rather involved, as the refined matching patches $\widehat{\mathcal{T}}_{\mathbf{a}}$ are not necessarily composed of elements sharing the vertex $\mathbf{a}$. For each non-hanging node $\mathbf{a} \in \mathcal{V}_{h}$, denote the set of vertices of $\widehat{\mathcal{T}}_{\mathbf{a}}$ where the hat function $\psi_{\mathbf{a}}$ is nonzero by $\widehat{\mathcal{V}}_{\mathbf{a}}$. For each $\hat{\mathbf{a}} \in \widehat{\mathcal{V}}_{\mathbf{a}}$, define the spaces

$$
\begin{array}{ll}
H_{*}^{1}\left(\omega_{\hat{\mathbf{a}}}\right):=\left\{v \in H^{1}\left(\omega_{\hat{\mathbf{a}}}\right) ;(v, 1)_{\omega_{\hat{\mathbf{a}}}}=0\right\}, & \hat{\mathbf{a}} \notin \overline{\Gamma_{\mathrm{D}}}, \\
H_{*}^{1}\left(\omega_{\hat{\mathbf{a}}}\right):=\left\{v \in H^{1}\left(\omega_{\hat{\mathbf{a}}}\right) ; v=0 \text { on } \partial \omega_{\hat{\mathbf{a}}} \cap \partial \Gamma_{\mathrm{D}}\right\}, & \mathbf{a} \in \overline{\Gamma_{\mathrm{D}}},
\end{array}
$$

where the subdomains $\omega_{\hat{\mathbf{a}}}$ are composed of the elements $\hat{K}$ of the refined matching patch $\widehat{\mathcal{T}}_{\mathbf{a}}$ which share the fine vertex $\hat{\mathbf{a}}$. Then, the Poincaré-Friedrichs inequality gives

$$
\|v\|_{\omega_{\hat{\mathbf{a}}}} \leq C_{\mathrm{PF}, \omega_{\hat{\mathbf{a}}}} h_{\omega_{\hat{\mathbf{a}}}}\|\nabla v\|_{\omega_{\hat{\mathbf{a}}}} \quad \forall v \in H_{*}^{1}\left(\omega_{\hat{\mathbf{a}}}\right)
$$


cf. Veeser and Verfürth [42] and the references therein. Similarly, the broken PoincaréFriedrichs inequality states that

$$
\|v\|_{\omega_{\hat{\mathbf{a}}}} \leq C_{\mathrm{bPF}, \omega_{\hat{\mathbf{a}}}} h_{\omega_{\hat{\mathbf{a}}}}\left(\|\nabla v\|_{\omega_{\hat{\mathbf{a}}}}+\left\{\sum_{\hat{e} \not \subset \overline{\Gamma_{\mathrm{N}}}, \hat{\mathbf{a}} \in \mathcal{V}_{\hat{e}}} h_{\hat{e}}^{-1}\left\|\Pi_{\hat{e}}^{0} \llbracket v \rrbracket\right\|_{\hat{e}}^{2}\right\}^{1 / 2}\right),
$$

for any piecewise (with respect to $\left.\widehat{\mathcal{T}}_{\mathbf{a}}\right) H^{1}$ function $v$ on $\omega_{\hat{\mathbf{a}}}$; the condition $(v, 1)_{\omega_{\hat{\mathbf{a}}}}=0$ needs to be imposed when $\hat{\mathbf{a}}$ lies in the interior of the domain $\Omega$ or in the interior of the Neumann boundary $\Gamma_{\mathrm{N}}$, see Brenner [7, Corollary 6.3 and Remark 1.1] and the references therein. Use the notation $\psi_{\hat{\mathbf{a}}}$ for the usual hat function on $\omega_{\hat{\mathbf{a}}}$ and define $C_{\text {cont,PF }}:=\max _{\mathbf{a} \in \mathcal{V}_{h}} \max _{\hat{\mathbf{a}} \in \widehat{\mathcal{V}}_{\mathbf{a}}}\left\{1+C_{\mathrm{PF}, \omega_{\hat{\mathbf{a}}}} h_{\omega_{\hat{\mathbf{a}}}}\left\|\nabla \psi_{\hat{\mathbf{a}}}\right\|_{\infty, \omega_{\hat{\mathbf{a}}}}\right\}$ and $C_{\text {cont,bPF }}:=$ $\max _{\mathbf{a} \in \mathcal{V}_{h}} \max _{\hat{\mathbf{a}} \in \widehat{\mathcal{V}}_{\mathbf{a}}}\left\{1+C_{\mathrm{bPF}, \omega_{\hat{\mathbf{a}}}} h_{\omega_{\hat{\mathbf{a}}}}\left\|\nabla \psi_{\hat{\mathbf{a}}}\right\|_{\infty, \omega_{\hat{\mathbf{a}}}}\right\}$. The constants $C_{\text {cont,PF }}$ and $C_{\text {cont,bPF }}$ only depend on the mesh regularity parameter $\kappa_{\widehat{\mathcal{T}}}$ of Assumption 2.1 and can be estimated, see the discussion in [21, proofs of Lemmas 3.12 and 3.13 and Section 4.3.2].

To state our local efficiency result, we need some more assumptions. First, we link the discrete gradient $\mathfrak{G}\left(u_{h}\right)$ to the broken gradient $\nabla u_{h}$ and the jumps of $u_{h}$ via

$$
\mathfrak{G}\left(u_{h}\right)=\nabla u_{h}-\vartheta \sum_{e \in \mathcal{E}_{h}} \mathfrak{l}_{e}\left(\llbracket u_{h} \rrbracket\right),
$$

where $\vartheta \in\{-1,0,1\}$ is a parameter (cf. (4.2) below), and the lifting operator $\mathfrak{l}_{e}$ : $L^{2}(e) \rightarrow\left[\mathbb{P}_{0}\left(\mathcal{T}_{e}\right)\right]^{2}$ is such that, cf. [17, Section 4.3$]$

$$
\left(\mathfrak{l}_{e}\left(\llbracket u_{h} \rrbracket\right), \mathbf{v}_{h}\right)_{\mathcal{T}_{e}}=\left\langle\left\{\left\{\mathbf{v}_{h}\right\} \cdot \mathbf{n}_{e}, \llbracket u_{h} \rrbracket\right\rangle_{e} \quad \forall \mathbf{v}_{h} \in\left[\mathbb{P}_{0}\left(\mathcal{T}_{e}\right)\right]^{2},\right.
$$

where $\mathcal{T}_{e}$ denotes the elements of the nonmatching mesh $\mathcal{T}_{h}$ sharing the edge $e$. Moreover, we assume henceforth that $u_{h}$ and $\mathfrak{G}\left(u_{h}\right)$ are piecewise polynomials in the sense

$$
u_{h} \in S_{h, \mathrm{p}}, \quad \mathfrak{G}\left(u_{h}\right) \in \nabla S_{h, \mathrm{p}},
$$

where $\mathrm{p}:=\left\{p_{K} \in \mathbb{N}, K \in \mathcal{T}_{h}\right\}$ denotes the elementwise polynomial degree and

$$
S_{h, \mathrm{p}}:=\left\{v_{h} \in H^{1}\left(\mathcal{T}_{h}\right) ;\left.v_{h}\right|_{K} \in \mathbb{R}_{p_{K}}(K) \quad \forall K \in \mathcal{T}_{h}\right\}
$$

Then the polynomial degrees $p_{\mathbf{a}}$ assigned to non-hanging vertices of Section 3.3 are

$$
p_{\mathbf{a}}:=\max _{K \in \mathcal{T}_{\mathbf{a}}} p_{K}, \quad \forall \mathbf{a} \in \mathcal{V}_{h}
$$

We will crucially use below polynomial-degree stability of mixed finite element methods in the form of [6, Theorem 7], based on [11, 12], that we employ on the fine matching patches sharing the vertex â partitioning $\omega_{\hat{\mathbf{a}}}$; we let $C_{\mathrm{st}}$ be the constant of $[21$, inequality $(3.40)]$, only depending on $\kappa_{\widehat{\mathcal{T}}}$.

Theorem 3.12 (Polynomial-degree-robust local efficiency for varying polynomial degree, inhomogeneous boundary conditions, mixed rectangular-triangular meshes, and meshes with hanging nodes). Let $u$ be the weak solution of (2.2), let $u_{h} \in S_{h, \mathrm{p}}$, and let $\mathfrak{G}\left(u_{h}\right) \in \nabla S_{h, \mathrm{p}}$. Assume that the local hat function orthogonality on the original patch domains $\omega_{\mathbf{a}}$ (3.6) holds and define by (3.30) the reconstruction polynomial degrees. Then, for $\boldsymbol{\sigma}_{\hat{h}}$ given by Definition 3.5, there holds, for all $K \in \mathcal{T}_{h}$,

$$
\begin{aligned}
\left\|\mathfrak{G}\left(u_{h}\right)+\boldsymbol{\sigma}_{\hat{h}}\right\|_{K} \leq & \sum_{\mathbf{a} \in \mathcal{V}_{K}}\left\{4\left(2 C_{\mathrm{st}} C_{\text {cont }, \mathrm{PF}}+C_{\mathrm{bPF}, \omega_{\mathbf{a}}} h_{\omega_{\mathbf{a}}} \max _{\hat{\mathbf{a}} \in \hat{\mathcal{V}}_{\mathbf{a}}}\left\|\nabla \psi_{\hat{\mathbf{a}}}\right\|_{\infty, \omega_{\hat{\mathbf{a}}}}\right)\right. \\
& \left.\times\left\|\nabla u-\mathfrak{G}\left(u_{h}\right)\right\|_{\omega_{\mathbf{a}}}+2 C_{\mathrm{st}}\left(\Delta_{f, \omega_{\mathbf{a}}}+\Delta_{\mathrm{N}, \omega_{\mathbf{a}}}\right)\right\}
\end{aligned}
$$


with the oscillation of the source term / inhomogeneous Neumann condition given by

$$
\begin{aligned}
\Delta_{f, \omega_{\hat{\mathbf{a}}}} & :=\left\{\sum_{\hat{K} \in \widehat{\mathcal{T}}_{\mathbf{a}}}\left(\frac{h_{\hat{K}}}{\pi}\left\|\psi_{\hat{\mathbf{a}}} f-\Pi_{Q_{\hat{h}}\left(\omega_{\hat{\mathbf{a}}}\right)}\left(\psi_{\hat{\mathbf{a}}} f\right)\right\|_{\hat{K}}\right)^{2}\right\}^{1 / 2}, \Delta_{f, \omega_{\mathbf{a}}}:=\left\{\sum_{\hat{\mathbf{a}} \in \hat{\mathcal{V}}_{\mathbf{a}}} \Delta_{f, \omega_{\hat{\mathbf{a}}}}^{2}\right\}^{1 / 2}, \\
\Delta_{\mathrm{N}, \omega_{\hat{\mathbf{a}}}} & :=\left\{\sum_{\hat{K} \in \widehat{\mathcal{T}}_{\mathbf{a}}}\left\{\sum_{\hat{e} \in \mathcal{E}_{\hat{K}}, \hat{e} \subset \overline{\Gamma_{\mathrm{N}}}}\left(C_{\mathrm{t}, \hat{K}, \hat{e}} h_{\hat{e}}^{1 / 2}\left\|\left(I-\Pi_{\mathbf{V}_{\hat{h}}\left(\omega_{\mathbf{a}}\right) \cdot \mathbf{n}}\right)\left(\psi_{\hat{\mathbf{a}}} \sigma_{\mathrm{N}}\right)\right\|_{\hat{e}}\right)\right\}^{2}\right\}^{1 / 2},
\end{aligned}
$$

and $\Delta_{\mathrm{N}, \omega_{\mathrm{a}}}:=\left\{\sum_{\hat{\mathrm{a}} \in \widehat{\mathcal{V}}_{\mathrm{a}}} \Delta_{\mathrm{N}, \omega_{\hat{\mathrm{a}}}}^{2}\right\}^{1 / 2}$.

Consider now Definition 3.8 of $s_{\hat{h}}$. Then, for all $K \in \mathcal{T}_{h}$, with the constant $C$ only depending on $\kappa_{\widehat{\mathcal{T}}}$, see [21, Section 4.3.2], the following holds:

$$
\begin{aligned}
\left\|\mathfrak{G}\left(u_{h}\right)-\nabla s_{\hat{h}}\right\|_{K} \leq & 4 C_{\mathrm{st}} C_{\text {cont }, \mathrm{bPF}} \sum_{\mathbf{a} \in \mathcal{V}_{K}}\left\|\nabla u-\mathfrak{G}\left(u_{h}\right)\right\|_{\omega_{\mathbf{a}}}+\sum_{\mathbf{a} \in \mathcal{V}_{K}} 2 C_{\mathrm{st}} \Delta_{\mathrm{D}, \omega_{\mathbf{a}}} \\
& +C \sum_{\mathbf{a} \in \mathcal{V}_{K}}\left\{\sum_{\hat{\mathbf{a}} \in \widehat{\mathcal{V}}_{\mathbf{a}}} \sum_{\hat{e} \not \subset \overline{\Gamma_{\mathrm{N}}}, \hat{\mathbf{a}} \in \mathcal{V}_{\hat{e}}} h_{\hat{e}}^{-1}\left\|\Pi_{\hat{e}}^{0} \llbracket u-u_{h} \rrbracket\right\|_{\hat{e}}^{2}\right\}^{1 / 2},
\end{aligned}
$$

with the inhomogeneous Dirichlet condition oscillation $\Delta_{\mathrm{D}, \omega_{\mathbf{a}}}:=\left\{\sum_{\hat{\mathbf{a}} \in \widehat{\mathcal{V}}_{\mathbf{a}}} \Delta_{\mathrm{D}, \omega_{\hat{\mathbf{a}}}}^{2}\right\}^{1 / 2}$,

$$
\Delta_{\mathrm{D}, \omega_{\hat{\mathbf{a}}}}:=\left\{\sum_{\hat{K} \in \widehat{\mathcal{T}}_{\mathbf{a}}}\left\{\sum_{\hat{e} \in \mathcal{E}_{\hat{K}}, \hat{e} \subset \overline{\Gamma_{\mathrm{D}}}}\left(C_{\mathrm{t}, \hat{K}, \hat{e}} h_{\hat{e}}^{1 / 2}\left\|\left(I-\Pi_{\mathbf{V}_{\hat{h}}\left(\omega_{\mathbf{a}}\right) \cdot \mathbf{n}}\right)\left(\nabla\left(\psi_{\hat{\mathbf{a}}} u_{\mathrm{D}}\right) \cdot \mathbf{t}_{\Omega}\right)\right\|_{\hat{e}}\right)\right\}^{2}\right\}^{1 / 2} .
$$

Proof. Fix an element $K$ of the original nonmatching mesh $\mathcal{T}_{h}$. Employing that $\left.\boldsymbol{\sigma}_{\hat{h}}\right|_{K}=\left.\sum_{\mathbf{a} \in \mathcal{V}_{K}} \boldsymbol{\varsigma}_{\hat{h}}^{\mathbf{a}}\right|_{K}$ from Definition 3.5, the partition of unity (2.3), and the triangle inequality, we infer that

$$
\left\|\mathfrak{G}\left(u_{h}\right)+\boldsymbol{\sigma}_{\hat{h}}\right\|_{K}=\left\|\left.\sum_{\mathbf{a} \in \mathcal{V}_{K}}\left(\psi_{\mathbf{a}} \mathfrak{G}\left(u_{h}\right)+\boldsymbol{\varsigma}_{\hat{h}}^{\mathbf{a}}\right)\right|_{K}\right\|_{K} \leq \sum_{\mathbf{a} \in \mathcal{V}_{K}}\left\|\psi_{\mathbf{a}} \mathfrak{G}\left(u_{h}\right)+\boldsymbol{\varsigma}_{\hat{h}}^{\mathbf{a}}\right\|_{\omega_{\mathbf{a}}}
$$

Similarly, for the potential reconstruction, it is enough to bound $\left\|\mathrm{R}_{\frac{\pi}{2}} \nabla\left(\psi_{\mathbf{a}} u_{h}\right)+\boldsymbol{\varsigma}_{\hat{h}}^{\mathbf{a}}\right\|_{\omega_{\mathbf{a}}}$ with $\boldsymbol{\varsigma}_{\hat{h}}^{\mathbf{a}}$ given by Definition 3.8. We reason in several steps:

1) The polynomial degree $p_{\mathbf{a}}$ of the reconstructions $\boldsymbol{\sigma}_{\hat{h}}$ and $s_{\hat{h}}$ on the refined matching patch $\widehat{\mathcal{T}}_{\mathbf{a}}$ is fixed by (3.30). This choice ensures that $p_{\mathbf{a}}$ is high enough for the conditions (3.41) and (3.43) of reference [21] to hold, so that we can rely on previous results of $[6,21]$ in this respect.

2) For interior vertices $\mathbf{a} \in \mathcal{V}_{h}^{\text {int }}$ on matching triangular or rectangular meshes, taking into account the previous point and the fact that the contributions on boundary edges $e \in \mathcal{E}_{K}^{\mathrm{N}}$ and $e \in \mathcal{E}_{K}^{\mathrm{D}}$ in (3.31) and (3.32), respectively, are discarded by the hat function $\psi_{\mathbf{a}}$, the assertions follow from [21, Theorem 3.17]. Indeed, now $\widehat{\mathcal{T}}_{\mathbf{a}}=\mathcal{T}_{\mathbf{a}}$ is only formed by (one-type) elements sharing the vertex $\mathbf{a}$, the set $\widehat{\mathcal{V}}_{\mathbf{a}}$ defined above only contains the vertex $\mathbf{a}$, so that $\omega_{\hat{\mathbf{a}}}=\omega_{\mathbf{a}}$ at all occurrences, and $\kappa_{\widehat{\mathcal{T}}}=\kappa_{\mathcal{T}}$.

3) For interior vertices $\mathbf{a} \in \mathcal{V}_{h}^{\text {int }}$ on matching mixed rectangular-triangular meshes, a careful inspection of the proof of [6, Theorem 7] implies (3.31) and (3.32) as in [21, Theorem 3.17]. The key is that the constructive proof goes over the individual elements and that the normal traces of Raviart-Thomas elements on both triangular and rectangular elements are polynomials of degree $p_{\mathbf{a}}$ on each edge, see Section 3.3, so that they can easily be matched. 
4) For boundary vertices $\mathbf{a} \in \mathcal{V}_{h}^{\text {ext }}$ on matching meshes, the inhomogeneous Neumann and Dirichlet boundary conditions can be treated as we show below in the general setting of nonmatching meshes.

5) We now move to treat the general situation for fluxes. Let $\mathbf{a} \in \mathcal{V}_{K}$, i.e., $\mathbf{a}$ is a non-hanging node of the original partition $\mathcal{T}_{h}$ such that the element $K$ lies in the patch $\mathcal{T}_{\mathbf{a}}$, see Assumption 2.1. Recall that $\mathcal{T}_{\mathbf{a}}$ is possibly nonmatching, with elements that do not share a. As the mesh $\widehat{\mathcal{T}}_{\mathbf{a}}$ of $\omega_{\mathbf{a}}$ is matching and respects the element type upon refinement, the hat function $\psi_{\mathbf{a}}$ on the original patch $\mathcal{T}_{\mathbf{a}}$ of the domain $\omega_{\mathbf{a}}$ can be expressed in terms of the fine hat functions $\psi_{\hat{\mathbf{a}}}$ in the form $\psi_{\mathbf{a}}=\sum_{\hat{\mathbf{a}} \in \widehat{\mathcal{V}}_{\mathbf{a}}} c_{\hat{\mathbf{a}}} \psi_{\hat{\mathbf{a}}}$ with coefficients $0<c_{\hat{\mathbf{a}}} \leq 1$. There is no orthogonality with respect to the fine hat functions $\psi_{\hat{\mathbf{a}}}$ on the fine patch domains $\omega_{\hat{\mathbf{a}}}$, in contrast to (3.6), so that we are lead to define the corresponding hat-orthogonality misfits on $\omega_{\hat{\mathbf{a}}}$ by

$$
\varrho_{\hat{\mathbf{a}}}:=\left\{\left(f \psi_{\hat{\mathbf{a}}}-\mathfrak{G}\left(u_{h}\right) \cdot \nabla \psi_{\hat{\mathbf{a}}}, 1\right)_{\omega_{\hat{\mathbf{a}}}}-\left\langle\psi_{\hat{\mathbf{a}}} \sigma_{\mathrm{N}}, 1\right\rangle_{\partial \omega_{\hat{\mathbf{a}}} \cap \Gamma_{\mathrm{N}}}\right\}\left|\omega_{\hat{\mathbf{a}}}\right|^{-1} .
$$

6) For each $\hat{\mathbf{a}} \in \widehat{\mathcal{V}}_{\mathbf{a}}$, we now identify a local problem posed in the space $H_{*}^{1}\left(\omega_{\hat{\mathbf{a}}}\right)$, see (3.23), and show that its solution is a lower bound on the energy error and data oscillation. It differs from that of [21, Lemma 3.12] by $\varrho_{\hat{a}}$ defined in (3.34) and by the inhomogeneous Neumann boundary condition. It reads: find $r_{\hat{\mathbf{a}}} \in H_{*}^{1}\left(\omega_{\hat{\mathbf{a}}}\right)$ such that

$$
\left(\nabla r_{\hat{\mathbf{a}}}, \nabla v\right)_{\omega_{\hat{\mathbf{a}}}}=-\left(\boldsymbol{\tau}_{h}^{\hat{\mathbf{a}}}, \nabla v\right)_{\omega_{\hat{\mathbf{a}}}}+\left(g^{\hat{\mathbf{a}}}, v\right)_{\omega_{\hat{\mathbf{a}}}}-\left\langle\Pi_{\mathbf{V}_{\hat{h}}\left(\omega_{\hat{\mathbf{a}}}\right) \cdot \mathbf{n}} \psi_{\hat{\mathbf{a}}} \sigma_{\mathrm{N}}, v\right\rangle_{\partial \omega_{\hat{\mathbf{a}}} \cap \Gamma_{\mathrm{N}}}-\left(\varrho_{\hat{\mathbf{a}}}, v\right)_{\omega_{\hat{\mathbf{a}}}},
$$

for all $v \in H_{*}^{1}\left(\omega_{\hat{\mathbf{a}}}\right)$, where $\tau_{h}^{\hat{\mathbf{a}}}:=\psi_{\hat{\mathbf{a}}} \mathfrak{G}\left(u_{h}\right)$ and $g^{\hat{\mathbf{a}}}:=\Pi_{Q_{\hat{h}}\left(\omega_{\hat{\mathbf{a}}}\right)}\left(\psi_{\hat{\mathbf{a}}} f\right)-\nabla \psi_{\hat{\mathbf{a}}} \cdot \mathfrak{G}\left(u_{h}\right)$. Note that the Neumann compatibility condition of this problem is satisfied owing to the definition (3.34) of $\varrho_{\hat{\mathbf{a}}}$ and owing to the use of $L^{2}(\Omega)$-projections. Note also that

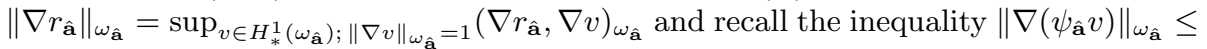
$C_{\text {cont,PF }}\|\nabla v\|_{\omega_{\hat{\mathbf{a}}}}$, cf. the beginning of the subsection. Then, proceeding as in [21, Lemma 3.12 and Theorem 3.17] and using the definition (2.2) of the weak solution, we infer that the contribution to $\left\|\nabla r_{\hat{\mathbf{a}}}\right\|_{\omega_{\hat{\mathbf{a}}}}$ by the first three terms on the right-hand side of (3.35) can be bounded by, with the above notation,

$$
C_{\text {cont }, \mathrm{PF}}\left\|\nabla u-\mathfrak{G}\left(u_{h}\right)\right\|_{\omega_{\hat{\mathbf{a}}}}+\Delta_{f, \omega_{\hat{\mathbf{a}}}}+\Delta_{\mathrm{N}, \omega_{\hat{\mathbf{a}}}} ;
$$

in particular, the Neumann boundary term is estimated as in the proof of Theorem 3.3. Finally, fix $v \in H_{*}^{1}\left(\omega_{\hat{\mathbf{a}}}\right)$ with $\|\nabla v\|_{\omega_{\hat{\mathbf{a}}}}=1$, employ the Cauchy-Schwarz inequality $\left|\left(\varrho_{\hat{\mathbf{a}}}, v\right)_{\omega_{\hat{\mathbf{a}}}}\right| \leq\left\|\varrho_{\hat{\mathbf{a}}}\right\|_{\omega_{\hat{\mathbf{a}}}}\|v\|_{\omega_{\hat{\mathbf{a}}}}$, and estimate, using (3.24),

$$
\begin{aligned}
\left\|\varrho_{\hat{\mathbf{a}}}\right\|_{\omega_{\hat{\mathbf{a}}}} & \leq\left|\left(\nabla u-\mathfrak{G}\left(u_{h}\right), \nabla \psi_{\hat{\mathbf{a}}}\right) \omega_{\hat{\mathbf{a}}}\right|\left|\omega_{\hat{\mathbf{a}}}\right|^{-1}\left|\omega_{\hat{\mathbf{a}}}\right|^{1 / 2} \leq\left\|\nabla u-\mathfrak{G}\left(u_{h}\right)\right\|_{\omega_{\hat{\mathbf{a}}}}\left\|\nabla \psi_{\hat{\mathbf{a}}}\right\|_{\infty, \omega_{\hat{\mathbf{a}}}}, \\
\|v\|_{\omega_{\hat{\mathbf{a}}}} & \leq C_{\mathrm{PF}, \omega_{\hat{\mathbf{a}}}} h_{\omega_{\hat{\mathbf{a}}}},
\end{aligned}
$$

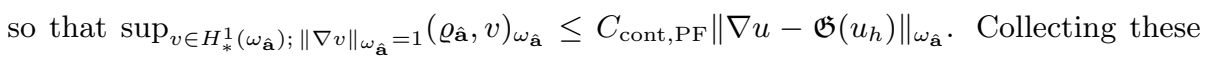
bounds, we arrive at

$$
\left\|\nabla r_{\hat{\mathbf{a}}}\right\|_{\omega_{\hat{\mathbf{a}}}} \leq 2 C_{\text {cont }, \mathrm{PF}}\left\|\nabla u-\mathfrak{G}\left(u_{h}\right)\right\|_{\omega_{\hat{\mathbf{a}}}}+\Delta_{f, \omega_{\hat{\mathbf{a}}}}+\Delta_{\mathrm{N}, \omega_{\hat{\mathbf{a}}}} .
$$

7) Define the mixed finite approximation of problems (3.35) for each $\hat{\mathbf{a}} \in \widehat{\mathcal{V}}_{\mathbf{a}}$ :

$$
\boldsymbol{\varsigma}_{1, \hat{h}}^{\hat{\mathbf{a}}}:=\arg \min _{\mathbf{v}_{\hat{h}} \in \mathbf{V}_{\hat{h}, \mathrm{~N}}^{\hat{\mathbf{a}}}, \nabla \cdot \mathbf{v}_{\hat{h}}=\Pi_{Q_{\hat{h}}^{\hat{\mathbf{a}}}}\left(\psi_{\hat{\mathbf{a}}} f-\nabla \psi_{\hat{\mathbf{a}}} \cdot \mathfrak{G}\left(u_{h}\right)-\varrho_{\hat{\mathbf{a}}}\right)}\left\|\psi_{\hat{\mathbf{a}}} \mathfrak{G}\left(u_{h}\right)+\mathbf{v}_{\hat{h}}\right\|_{\omega_{\hat{\mathbf{a}}}},
$$

similarly to (3.13), but on the fine patch domains $\omega_{\hat{\mathbf{a}}}$. As all the data of (3.35) are piecewise polynomials, the stability of [6, Theorem 7] (see also [21, Corollary 3.16]) and step 3) immediately yield

$$
\left\|\psi_{\hat{\mathbf{a}}} \mathfrak{G}\left(u_{h}\right)+\varsigma_{1, \hat{h}}^{\hat{\mathbf{a}}}\right\|_{\omega_{\hat{\mathbf{a}}}} \leq C_{\mathrm{st}}\left\|\nabla r_{\hat{\mathbf{a}}}\right\|_{\omega_{\hat{\mathbf{a}}}} .
$$


8) Define $\boldsymbol{\varsigma}_{1, \hat{h}}^{\mathbf{a}}:=\sum_{\hat{\mathbf{a}} \in \widehat{\mathcal{V}}_{\mathbf{a}}} c_{\hat{\mathbf{a}}} \boldsymbol{\varsigma}_{1, \hat{h}}^{\hat{\mathbf{a}}}$. Using $\psi_{\mathbf{a}}=\sum_{\hat{\mathbf{a}} \in \widehat{\mathcal{V}}_{\mathbf{a}}} c_{\hat{\mathbf{a}}} \psi_{\hat{\mathbf{a}}}$, summing as in [21, Lemma 3.22] (the considered elements have at most 4 edges), and employing $c_{\hat{\mathbf{a}}} \leq 1$,

$$
\left\|\psi_{\mathbf{a}} \mathfrak{G}\left(u_{h}\right)+\boldsymbol{\varsigma}_{1, \hat{h}}^{\mathbf{a}}\right\|_{\omega_{\mathbf{a}}}^{2}=\sum_{\hat{K} \in \widehat{\mathcal{T}}_{\mathbf{a}}}\left\|\left.\sum_{\hat{\mathbf{a}} \in \mathcal{V}_{\hat{K}}} c_{\hat{\mathbf{a}}}\left(\psi_{\hat{\mathbf{a}}} \mathfrak{G}\left(u_{h}\right)+\boldsymbol{\varsigma}_{1, \hat{h}}^{\hat{\mathbf{a}}}\right)\right|_{\hat{K}}\right\|_{\hat{K}}^{2} \leq 4 \sum_{\hat{\mathbf{a}} \in \hat{\mathcal{V}}_{\mathbf{a}}}\left\|\psi_{\hat{\mathbf{a}}} \mathfrak{G}\left(u_{h}\right)+\boldsymbol{\varsigma}_{1, \hat{h}}^{\hat{\mathbf{a}}}\right\|_{\omega_{\hat{\mathbf{a}}}}^{2} ;
$$

here $\mathcal{V}_{\hat{K}}$ simply stands for the vertices of the fine element $\hat{K}$. Combining this estimate with the bounds (3.37) and (3.36), we arrive at

$$
\begin{aligned}
\left\|\psi_{\mathbf{a}} \mathfrak{G}\left(u_{h}\right)+\boldsymbol{\varsigma}_{1, \hat{h}}^{\mathbf{a}}\right\|_{\omega_{\mathbf{a}}} & \leq 2 C_{\text {st }}\left\{\sum_{\hat{\mathbf{a}} \in \hat{\mathcal{V}}_{\mathbf{a}}}\left(2 C_{\text {cont }, \mathrm{PF}}\left\|\nabla u-\mathfrak{G}\left(u_{h}\right)\right\|_{\omega_{\hat{\mathbf{a}}}}+\Delta_{f, \omega_{\hat{\mathbf{a}}}}+\Delta_{\mathrm{N}, \omega_{\hat{\mathbf{a}}}}\right)^{2}\right\}^{1 / 2} \\
& \leq 2 C_{\mathrm{st}}\left(4 C_{\mathrm{cont}, \mathrm{PF}}\left\|\nabla u-\mathfrak{G}\left(u_{h}\right)\right\|_{\omega_{\mathbf{a}}}+\Delta_{f, \omega_{\mathbf{a}}}+\Delta_{\mathrm{N}, \omega_{\mathbf{a}}}\right) .
\end{aligned}
$$

9) Because of its divergence, the function $\boldsymbol{\varsigma}_{1, \hat{h}}^{\mathbf{a}}$ cannot be used as the function $\mathbf{v}_{\hat{h}}$ in (3.13). To fix this, we now construct a convenient $\boldsymbol{\varsigma}_{2, \hat{h}}^{\mathbf{a}}$. Define first a $\widehat{\mathcal{T}}_{\mathbf{a}^{-}}$ piecewise-constant function $\varrho_{\mathbf{a}}:=\left.\sum_{\hat{\mathbf{a}} \in \widehat{\mathcal{V}}_{\mathbf{a}}} c_{\hat{\mathbf{a}}} \varrho_{\hat{\mathbf{a}}} 1\right|_{\omega_{\hat{\mathbf{a}}}}$. We next check that, from (3.34), $\psi_{\mathbf{a}}=\sum_{\hat{\mathbf{a}} \in \widehat{\mathcal{V}}_{\mathbf{a}}} c_{\hat{\mathbf{a}}} \psi_{\hat{\mathbf{a}}}$, and (crucially) (3.6), $\left(\varrho_{\mathbf{a}}, 1\right)_{\omega_{\mathbf{a}}}=\sum_{\hat{\mathbf{a}} \in \widehat{\mathcal{V}}_{\mathbf{a}}} c_{\hat{\mathbf{a}}} \varrho_{\hat{\mathbf{a}}}\left|\omega_{\hat{\mathbf{a}}}\right|=\left(f \psi_{\mathbf{a}}-\right.$ $\left.\mathfrak{G}\left(u_{h}\right) \cdot \nabla \psi_{\mathbf{a}}, 1\right)_{\omega_{\mathbf{a}}}-\left\langle\psi_{\mathbf{a}} \sigma_{\mathrm{N}}, 1\right\rangle_{\partial \omega_{\mathbf{a}} \cap \Gamma_{\mathrm{N}}}=0$. Thus the constrained minimization (mixed finite element local Neumann problem)

$$
\boldsymbol{\varsigma}_{2, \hat{h}}^{\mathbf{a}}:=\arg \min _{\mathbf{v}_{\hat{h}} \in \mathbf{V}_{\hat{h}}^{0}\left(\omega_{\mathbf{a}}\right) ; \mathbf{v}_{\hat{h}} \cdot \mathbf{n}_{\omega_{\mathbf{a}}}=0, \nabla \cdot \mathbf{v}_{\hat{h}}=\varrho_{\mathbf{a}}}\left\|\mathbf{v}_{\hat{h}}\right\|_{\omega_{\mathbf{a}}}
$$

is well-posed (here we importantly only use the lowest order space $\mathbf{V}_{\hat{h}}^{0}\left(\omega_{\mathbf{a}}\right)$ as $\varrho_{\mathbf{a}}$ is piecewise constant). Its stability (cf., e.g., [44, Theorem 5.9]) gives

$$
\left\|\varsigma_{2, \hat{h}}^{\mathbf{a}}\right\|_{\omega_{\mathbf{a}}} \leq C_{\mathrm{bPF}, \omega_{\mathbf{a}}} h_{\omega_{\mathbf{a}}}\left\|\varrho_{\mathbf{a}}\right\|_{\omega_{\mathbf{a}}}
$$

Proceeding as in steps 8) and 6), we infer that

$$
\left\|\varrho_{\mathbf{a}}\right\|_{\omega_{\mathbf{a}}}^{2} \leq 4 \sum_{\hat{\mathbf{a}} \in \widehat{\mathcal{V}}_{\mathbf{a}}}\left\|\varrho_{\hat{\mathbf{a}}}\right\|_{\omega_{\hat{\mathbf{a}}}}^{2} \leq 4 \max _{\hat{\mathbf{a}} \in \widehat{\mathcal{V}}_{\mathbf{a}}}\left\|\nabla \psi_{\hat{\mathbf{a}}}\right\|_{\infty, \omega_{\hat{\mathbf{a}}}}^{2} \sum_{\hat{\mathbf{a}} \in \widehat{\mathcal{V}}_{\mathbf{a}}}\left\|\nabla u-\mathfrak{G}\left(u_{h}\right)\right\|_{\omega_{\hat{\mathbf{a}}}}^{2},
$$

so that altogether

$$
\left\|\boldsymbol{\varsigma}_{2, \hat{h}}^{\mathbf{a}}\right\| \omega_{\omega_{\mathbf{a}}} \leq 4 C_{\mathrm{bPF}, \omega_{\mathbf{a}}} h_{\omega_{\mathbf{a}}} \max _{\hat{\mathbf{a}} \in \hat{\mathcal{V}}_{\mathbf{a}}}\left\|\nabla \psi_{\hat{\mathbf{a}}}\right\|_{\infty, \omega_{\hat{\mathbf{a}}}}\left\|\nabla u-\mathfrak{G}\left(u_{h}\right)\right\|_{\omega_{\mathbf{a}}}
$$

10) We now notice that $\boldsymbol{\varsigma}_{1, \hat{h}}^{\mathbf{a}}+\boldsymbol{\varsigma}_{2, \hat{h}}^{\mathbf{a}}$ is an admissible function $\mathbf{v}_{\hat{h}}$ in (3.13), so that $\left\|\psi_{\mathbf{a}} \mathfrak{G}\left(u_{h}\right)+\varsigma_{1, \hat{h}}^{\mathbf{a}}+\boldsymbol{\varsigma}_{2, \hat{h}}^{\mathbf{a}}\right\|_{\omega_{\mathbf{a}}} \leq\left\|\psi_{\mathbf{a}} \mathfrak{G}\left(u_{h}\right)+\boldsymbol{\varsigma}_{1, \hat{h}}^{\mathbf{a}}\right\|_{\omega_{\mathbf{a}}}+\left\|\boldsymbol{\varsigma}_{2, \hat{h}}^{\mathbf{a}}\right\|_{\omega_{\mathbf{a}}}$, and the bounds (3.38) and (3.40) together with (3.33) yield the assertion (3.31).

11) As for (3.33) from the beginning of the proof, we now need to estimate $\left\|\mathrm{R}_{\frac{\pi}{2}} \nabla\left(\psi_{\mathbf{a}} u_{h}\right)+\boldsymbol{\varsigma}_{\hat{h}}^{\mathbf{a}}\right\|_{\omega_{\mathbf{a}}}$ for a non-hanging vertex $\mathbf{a} \in \mathcal{V}_{h}$ of the original mesh $\mathcal{T}_{h}$ on the patch $\omega_{\mathbf{a}}$ defined in Assumption 2.1. Note in particular that the Dirichlet boundary condition on $\Gamma_{D}$ appears here as a Neumann boundary condition on $\partial \omega_{\mathbf{a}} \cap \Gamma_{\mathrm{D}}$, see (3.16). Following [21, Lemma 3.13], for each vertex $\hat{\mathbf{a}} \in \widehat{\mathcal{V}}_{\mathbf{a}}$ of the refined matching patch $\widehat{\mathcal{T}}_{\mathbf{a}}$ where $\psi_{\mathbf{a}}$ is nonzero, we introduce the problem: find $r_{\hat{\mathbf{a}}} \in H_{\mathrm{b}}^{1}\left(\widehat{\mathcal{T}}_{\mathbf{a}}\right)$ such that

$$
\left(\nabla r_{\hat{\mathbf{a}}}, \nabla v\right)_{\omega_{\hat{\mathbf{a}}}}=-\left(\boldsymbol{\tau}_{h}^{\hat{\mathbf{a}}}, \nabla v\right)_{\omega_{\hat{\mathbf{a}}}}+\left(g^{\hat{\mathbf{a}}}, v\right)_{\omega_{\hat{\mathbf{a}}}}-\left\langle g_{\mathrm{D}}^{\mathbf{a}}, v\right\rangle_{\partial \omega_{\hat{\mathbf{a}}} \cap \Gamma_{\mathrm{D}}} \quad \forall v \in H_{b}^{1}\left(\widehat{\mathcal{T}}_{\mathbf{a}}\right),
$$


with $g_{\mathrm{D}}^{\mathbf{a}}:=\nabla\left(\psi_{\hat{\mathbf{a}}} u_{\mathrm{D}}\right) \cdot \mathbf{t}_{\Omega}, \tau_{h}^{\hat{\mathbf{a}}}:=\mathrm{R}_{\frac{\pi}{2}} \nabla\left(\psi_{\hat{\mathbf{a}}} u_{h}\right)$, and $g^{\hat{\mathbf{a}}}:=0$, and with

$$
\begin{array}{lll}
H_{b}^{1}\left(\widehat{\mathcal{T}}_{\mathbf{a}}\right):=\left\{v \in H^{1}\left(\omega_{\hat{\mathbf{a}}}\right) ;(v, 1)_{\omega_{\hat{\mathbf{a}}}}=0\right\}, & \hat{\mathbf{a}} \notin \overline{\Gamma_{\mathrm{N}}} \\
H_{b}^{1}\left(\widehat{\mathcal{T}}_{\mathbf{a}}\right):=\left\{v \in H^{1}\left(\omega_{\hat{\mathbf{a}}}\right) ; v=0 \text { on } \partial \omega_{\hat{\mathbf{a}}} \cap \Gamma_{\mathrm{N}}\right\}, & \hat{\mathbf{a}} \in \overline{\Gamma_{\mathrm{N}}}
\end{array}
$$

We are again lead to study the above problem with a polynomial Neumann term given by $\tilde{g}_{\mathrm{D}}^{\mathbf{a}}:=\Pi_{\mathbf{V}_{\hat{h}}\left(\omega_{\hat{\mathbf{a}}}\right) \cdot \mathbf{n}}\left(\nabla\left(\psi_{\hat{\mathbf{a}}} u_{\mathrm{D}}\right) \cdot \mathbf{t}_{\Omega}\right)$. We denote its solution by $\tilde{r}_{\hat{\mathbf{a}}}$ and need to bound

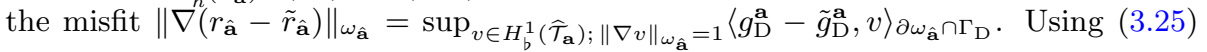
and proceeding as in [21, Section 4.3.2], we altogether obtain

$$
\left\|\nabla \tilde{r}_{\hat{\mathbf{a}}}\right\|_{\omega_{\hat{\mathbf{a}}}} \leq C_{\text {cont, bPF }}\left(\left\|\nabla\left(u-u_{h}\right)\right\|_{\omega_{\hat{\mathbf{a}}}}+\left\{\sum_{\hat{e} \not \subset \overline{\Gamma_{\mathrm{N}}}, \hat{\mathbf{a}} \in \mathcal{V}_{\hat{e}}} h_{\hat{e}}^{-1}\left\|\Pi_{\hat{e}}^{0} \llbracket u-u_{h} \rrbracket\right\|_{\hat{e}}^{2}\right\}^{1 / 2}\right)+\Delta_{\mathrm{D}, \omega_{\hat{\mathbf{a}}}},
$$

whereas [6, Theorem 7] again implies $\left\|\mathrm{R}_{\frac{\pi}{2}} \nabla\left(\psi_{\hat{\mathbf{a}}} u_{h}\right)+\varsigma_{\hat{h}}^{\hat{\mathbf{a}}}\right\|_{\omega_{\hat{\mathbf{a}}}} \leq C_{\mathrm{st}}\left\|\nabla \tilde{r}_{\hat{\mathbf{a}}}\right\|_{\omega_{\hat{\mathbf{a}}}}$ for the reconstruction $\boldsymbol{\varsigma}_{\hat{h}}^{\hat{a}}$ on the fine usual patch domain $\omega_{\hat{\mathbf{a}}}$. Proceeding as in (3.38) and employing (3.26) allows us to conclude that (3.32) holds, as in [21, Section 4.3.2].

\section{Discretization, implementation, and asymptotic exactness under uniform refinement}

This section briefly outlines the interior penalty discontinuous Galerkin method, discusses some implementation aspects of the local Raviart-Thomas spaces, and presents a numerical illustration of asymptotic exactness under uniform refinement for a smooth solution.

\subsection{Discretization by the interior penalty discontinuous Galerkin method}

Consider the interior penalty discontinuous Galerkin (IPDG) method: find $u_{h} \in S_{h, \mathrm{p}}$ (defined by (3.29)) such that

$$
a_{h}\left(u_{h}, v_{h}\right)=l_{h}\left(v_{h}\right) \quad \forall v_{h} \in S_{h, \mathrm{p}}
$$

where

$$
\begin{aligned}
a_{h}\left(u_{h}, v_{h}\right):= & \sum_{K \in \mathcal{T}_{h}}\left(\nabla u_{h}, \nabla v_{h}\right)_{K}+\sum_{e \in \mathcal{E}_{h}^{\text {int }} \cup \mathcal{E}_{h}^{\text {ext }, \mathrm{D}}}\left\langle\alpha h_{e}^{-1} \llbracket u_{h} \rrbracket, \llbracket v_{h} \rrbracket\right\rangle_{e} \\
& -\sum_{e \in \mathcal{E}_{h}^{\text {int }} \cup \mathcal{E}_{h}^{\text {ext }, \mathrm{D}}}\left\{\left\langle\left\{\left\{\nabla u_{h}\right\} \cdot \mathbf{n}_{e}, \llbracket v_{h} \rrbracket\right\rangle_{e}+\vartheta\left\langle\left\{\nabla \nabla v_{h}\right\}\right\} \cdot \mathbf{n}_{e}, \llbracket u_{h} \rrbracket\right\rangle_{e}\right\} \\
l_{h}\left(v_{h}\right):= & \left(f, v_{h}\right)+\sum_{e \in \mathcal{E}_{h}^{\text {ext }, \mathrm{D}}}\left(\left\langle\alpha h_{e}^{-1} u_{\mathrm{D}}, v_{h}\right\rangle_{e}-\vartheta\left\langle\nabla v_{h} \cdot \mathbf{n}_{e}, u_{\mathrm{D}}\right\rangle_{e}\right)-\left\langle\sigma_{\mathrm{N}}, v_{h}\right\rangle_{\Gamma_{\mathrm{N}}},
\end{aligned}
$$

$\{\{\}\}$ and $\llbracket \cdot \rrbracket$ denote the mean value and jump operator, respectively, see Section 2, $\alpha$ is a positive penalty parameter, and $\vartheta \in\{-1,0,1\}$ corresponds respectively to the nonsymmetric (NIPG), incomplete (IIPG), and symmetric (SIPG) versions of the method; $\vartheta$ is the parameter considered in (3.26). Taking $\psi_{\mathbf{a}} \in S_{h, \mathrm{p}}$ as the test function in (4.2) for the vertices $\mathbf{a} \in \mathcal{V}_{h} \backslash \mathcal{V}_{h}^{\text {ext,D }}$ and using the definition (3.26) of the discrete gradient, we infer the hat-function orthogonality condition (3.6). 


\begin{tabular}{|c|c|c|c|}
\hline$p$ & original & cell orth. & cell and edge orth. \\
\hline 1 & $2.24 \mathrm{E}+01$ & $2.77 \mathrm{E}+01$ & $3.60 \mathrm{E}+01$ \\
2 & $1.78 \mathrm{E}+02$ & $1.09 \mathrm{E}+02$ & $1.07 \mathrm{E}+02$ \\
3 & $4.74 \mathrm{E}+03$ & $2.44 \mathrm{E}+02$ & $2.02 \mathrm{E}+02$ \\
4 & $1.42 \mathrm{E}+05$ & $5.41 \mathrm{E}+02$ & $3.93 \mathrm{E}+02$ \\
5 & $4.66 \mathrm{E}+06$ & $1.10 \mathrm{E}+03$ & $6.24 \mathrm{E}+02$ \\
6 & $1.46 \mathrm{E}+08$ & $2.34 \mathrm{E}+03$ & $9.63 \mathrm{E}+02$ \\
7 & $4.43 \mathrm{E}+09$ & $5.18 \mathrm{E}+03$ & $1.16 \mathrm{E}+03$ \\
\hline
\end{tabular}

Tab. 1: Mass matrix condition number for the local mixed finite element problems with various choices of the basis functions as a function of polynomial degree $p$, generic shape-regular patch

An important issue with interior penalty discontinuous Galerkin methods is the choice of the penalty parameter $\alpha$. Typical choices, supported theoretically and numerically, are $\alpha=O\left(p^{2}\right)$ for the symmetric variant and a $p$-independent choice $\alpha>0$ for the nonsymmetric one. Numerical evidence indicates that a $p$-independent choice for $\alpha$ is also possible for the incomplete version.

The IPDG discretization leads to a linear algebraic system which can be written in the block-matrix form (one block-row for each $K \in \mathcal{T}_{h}$ )

$$
\mathbb{A}_{K, K} \mathbf{u}_{K}+\sum_{K^{\prime} \in N(K)} \mathbb{A}_{K, K^{\prime}} \mathbf{u}_{K^{\prime}}=\mathbf{f}_{K} \quad \forall K \in \mathcal{T}_{h},
$$

where $\mathbb{A}_{K, K}$ are diagonal blocks of size $m_{K} \times m_{K}, m_{K}$ is the number of degrees of freedom attached to the element $K \in \mathcal{T}_{h}$ given by $m_{K}=\left(p_{K}+1\right)\left(p_{K}+2\right) / 2$ (on triangles). Moreover, $N(K)$ is the set of neighbouring elements sharing an edge with $K$, and $\mathbb{A}_{K, K^{\prime}}$, for all $K^{\prime} \in N(K)$, are the corresponding off-diagonal blocks. Finally, $\mathbf{u}_{K} \in \mathbb{R}^{m_{K}}$ is the vector of the basis coeficients of the approximate solution $\left.u_{h}\right|_{K}$, $K \in \mathcal{T}_{h}$, and $\mathbf{f}_{K} \in \mathbb{R}^{m_{K}}, K \in \mathcal{T}_{h}$, are the corresponding blocks of the right-hand side.

\subsection{Implementation of the mixed finite element solves}

The patchwise mixed finite element problems (3.7) and (3.14) are solved using the Schur complement and a direct solver. When the polynomial degree grows, the choice of basis functions is important so as to tame the growth of the condition number of the local Raviart-Thomas mass matrices. Starting with the usual shape functions that constitute the dual basis of the canonical degrees of freedom, significant improvements in the condition number of the mass matrix are observed if a Gram-Schmidt process is applied to $L^{2}$-orthogonalize the shape functions attached to cell-based degrees of freedom. A further marginal reduction of the condition number is achieved if further linear combinations are formed to orthogonalize cell- and edge-based shape functions. Elemental results are presented in Table 1. We observe that the cell-based $L^{2}$-orthogonalization already leads to a reasonable conditioning.

\subsection{Uniform mesh and polynomial degree refinement for a smooth solution}

We first illustrate the behavior of the derived estimates for a smooth solution and matching triangular grids. We consider $\Omega=(0,1) \times(0,1)$ and take $u\left(x_{1}, x_{2}\right)=$ $\sin \left(2 \pi x_{1}\right) \sin \left(2 \pi x_{2}\right)$. Here a given sequence of four unstructured non-nested grids 


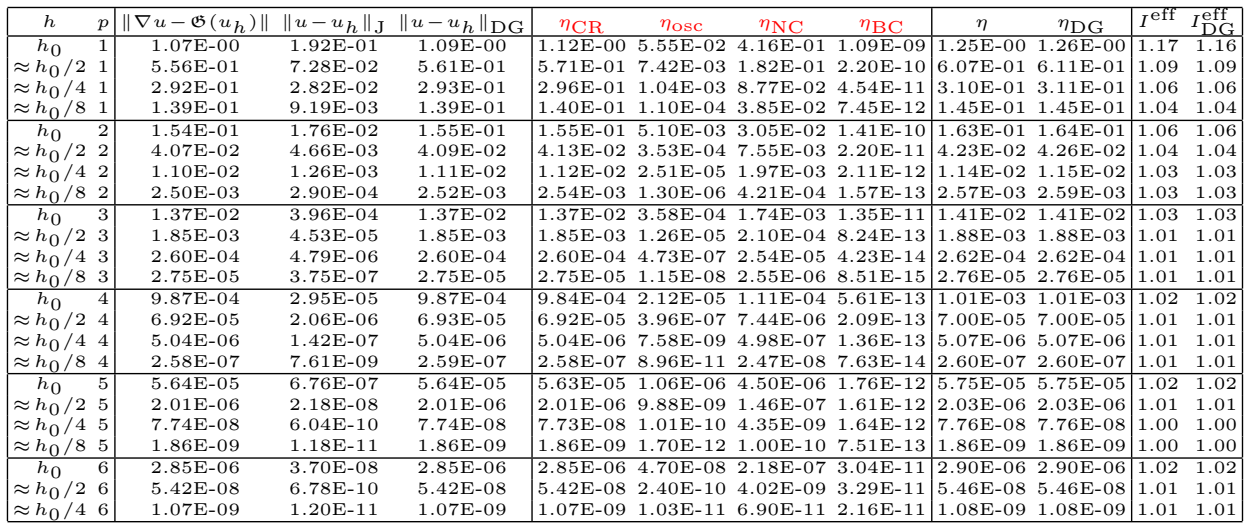

Tab. 2: Errors and estimates for a smooth solution and matching triangular grids, SIPG method

is considered, with SIPG and NIPG methods and polynomial approximations up to order 6 (similar results on uniformly refined nested grids in the IIPG case were already presented in [21, Section 5]). The penalty parameter was chosen as $\alpha=5 p^{2}$ for SIPG and $\alpha=1$ for NIPG. The results are presented in Tables 2-3, where $\left\|u-u_{h}\right\|_{J}^{2}:=\sum_{e \in \mathcal{E}_{h}} h_{e}^{-1}\left\|\Pi_{e}^{0} \llbracket u-u_{h} \rrbracket\right\|_{e}^{2}$ is the jump seminorm and $\left\|u-u_{h}\right\|_{\mathrm{DG}}^{2}:=$ $\left\|\nabla u-\mathfrak{G}\left(u_{h}\right)\right\|^{2}+\left\|u-u_{h}\right\|_{\mathrm{J}}^{2}$ is the DG norm. The estimator $\eta$ and its components are given by Theorem 3.3; the full DG estimator is $\eta_{\mathrm{DG}}^{2}:=\eta^{2}+\left\|u-u_{h}\right\|_{\mathrm{J}}^{2}$. The tables also report the effectivity indices $I^{\text {eff }}:=\frac{\eta}{\left\|\nabla\left(u-u_{h}\right)\right\|}$ and $I_{\mathrm{DG}}^{\text {eff }}:=\frac{\eta_{\mathrm{DG}}}{\left\|u-u_{h}\right\|_{\mathrm{DG}}}$. Asymptotic exactness is observed for SIPG, as well as for NIPG with odd polynomial degrees, similarly to [21, Section 5]. Even polynomial degrees for NIPG lead to effectivity indices close to one, the more so as the polynomial degree is increased.

\section{$5 \quad h p$-adaptive strategies}

Theoretical and numerical results (e.g., [1, 5, 13, 19, 22, 23, 24, 25, 28, 29, 31, 37, 38, $39])$ show that $h p$-adaptivity can lead to an exponential rate of the convergence with respect to the number of degrees of freedom (DoF) in the sense that, in two dimensions,

$$
\left\|\nabla\left(u-u_{h}\right)\right\| \approx C \exp \left(-b \operatorname{DoF}^{1 / 3}\right)
$$

where $C>0$ and $b>0$ are constants independent of DoF. In the context of the interior penalty discontinuous Galerkin method from Section 4.1, DoF is the dimension of the space $S_{h, \mathrm{p}}$. The $h p$-adaptive algorithm that we consider reads as follows, where $\varpi>0$ is a user-prescribed tolerance:

1. Compute $u_{h} \in S_{h p}$ and evaluate the equilibrated flux a posteriori error estimates $\eta_{K}$ of Theorem 3.3 for all the elements $K$ of the current mesh $\mathcal{T}_{h}$;

2. If $\eta^{2}=\sum_{K \in \mathcal{T}_{h}} \eta_{K}^{2} \leq \varpi^{2}$, then stop to computation, else sort $10 \%$ of the elements having the largest $\eta_{K}$ and mark them for refinement; 


\begin{tabular}{|c|c|c|c|c|c|c|c|c|c|c|c|c|}
\hline$h$ & $p$ & $\left\|\nabla u-\mathfrak{G}\left(u_{h}\right)\right\|$ & $\left\|u-u_{h}\right\|_{\mathrm{J}}$ & $\left\|u-u_{h}\right\|_{\mathrm{DG}}$ & & $\eta_{\mathrm{O} s}$ & & & $\eta$ & & $I^{\mathrm{eff}}$ & $I_{\mathrm{DG}}^{\mathrm{erf}}$ \\
\hline$\overline{h_{0}}$ & 1 & $1.08 \mathrm{E}-00$ & $1.69 \mathrm{E}-01$ & $1.09 \mathrm{E}-00$ & & $5.55 \mathrm{E}-02$ & $.98 \mathrm{E}-01$ & $1.09 \mathrm{E}-09$ & $1.17 \mathrm{E}-00$ & $1.18 \mathrm{E}-00$ & 1.09 & \\
\hline$h_{0} / 2$ & & 5 & -02 & & -01 & 7.42 & $3.75 \mathrm{E}-01$ & $2.20 \mathrm{E}-10$ & $5.66 \mathrm{E}-01$ & E-01 & 1.03 & \\
\hline & & $2.84 \mathrm{E}-01$ & $34 \mathrm{E}-02$ & $36 \mathrm{E}-01$ & $8 \mathrm{E}-01$ & $1.04 \mathrm{E}-03$ & $1.86 \mathrm{E}-01$ & $4.54 \mathrm{E}-11$ & $2.87 \mathrm{E}-01$ & $2.89 \mathrm{E}-01$ & 1.01 & \\
\hline$h_{0} / 8$ & & 1.3 & & & & & & & & & 1.01 & \\
\hline$h_{0}$ & 2 & & 22 & & & 5.10 & 1.7 & & $2.24 \mathrm{~F}$ & & 1.36 & 33 \\
\hline & & & & & & & & & $6.01 \mathrm{I}$ & & 41 & 1.38 \\
\hline & & & & & & & & & & & & \\
\hline$h_{0} / 82$ & 2 & 0 & 04 & & 03 & 1.301 & 2.991 & $1.56 \mathrm{E}-13$ & $3.74 \mathrm{E}-03$ & $3.82 \mathrm{E}-03$ & 1.45 & 1.42 \\
\hline & & & & & & & & & & & 1.08 & 1.08 \\
\hline$\sim w_{b}$ & & & & & & & & & & & & 1.05 \\
\hline$h_{\mathrm{c}}$ & & & & & & & & 4.2 & 04 & 04 & 1.03 & 1.03 \\
\hline$\approx h_{0} / 8$ & & & & & & & & & & & 1.02 & 02 \\
\hline & & & & & & & & & & & 1.11 & 1.11 \\
\hline & & & & & & & & & & & 1.11 & \\
\hline & & & & & & & & & & & 1.11 & 11 \\
\hline$\approx h_{0} / 8$ & & & & & 7 & & & & & & 1.12 & 1.12 \\
\hline & & & & & & & & & & & 5 & 705 \\
\hline & & & & & & & & & & & & 0, \\
\hline$\sim n$ & & & & & & & & & & & 2 & 1.02 \\
\hline$\approx h_{0} / 8$ & & & & & & & & & & 09 & 1.02 & 1 \\
\hline & & & & & & & & & & & 1.07 & $\overline{1.0}$ \\
\hline & & & & & & & & & & & 1.06 & 1.0 \\
\hline & & 1.20 & & 99 & -09 & $03 \mathrm{E}-11$ & $1 \mathrm{E}-10$ & $.06 \mathrm{E}-11$ & $.28 \mathrm{E}-09$ & $1.28 \mathrm{E}-09$ & 1.07 & 1.07 \\
\hline
\end{tabular}

Tab. 3: Errors and estimates for a smooth solution and matching triangular grids , NIPG method

3. For each marked element, decide between $h$ - and $p$-refinement; for $p$-refinement increase $p_{K}$ by 1 , and for $h$-refinement split $K$ into 4 similar subelements (hanging nodes typically arise) and go back to step 1.

An $h p$-adaptive algorithm thus combines an $h p$-a-posteriori error estimate (Step 1), a marking strategy (Step 2), and an $h p$-decision criterion (Step 3). The rest of this section is devoted to the description of three $h p$-decision criteria to be compared in our numerical experiments in Section 6. Two are essentially drawn from the literature and one is novel. Before we do this, we present a common ingredient to all of them, namely a projection of the discrete solution onto the lower-polynomial-degree space $S_{h, \mathrm{p}-1}$.

\subsection{Projection onto the lower-polynomial-degree space}

We define the projection $u_{h}^{\mathrm{p}-1}$ of $u_{h}$ onto the lower-polynomial-degree space $S_{h, \mathrm{p}-1}$ by solving a local problem in each element $K \in \mathcal{T}_{h}$. For each $K \in \mathcal{T}_{h}$, we set $\left.u_{h}^{\mathrm{p}-1}\right|_{K}:=$ $\left.\tilde{u}^{K}\right|_{K}$ where $\left.\tilde{u}^{K}\right|_{K^{\prime}}:=\left.u_{h}\right|_{K^{\prime}}$ for all $K^{\prime} \in \mathcal{T}_{h}$ with $K^{\prime} \neq K$, while $\left.\tilde{u}^{K}\right|_{K} \in \mathbb{R}_{p_{K}-1}(K)$ is determined by solving $a_{h}\left(\tilde{u}^{K}, v_{h}\right)=l_{h}\left(v_{h}\right)$ for all $v_{h} \in \mathbb{R}_{p_{K}-1}(K)$, where $a_{h}$ and $l_{h}$ are the forms defined in (4.2). In practice, we consider local hierarchical orthogonal polynomials bases of $S_{h, \mathrm{p}}$. Let $\Pi_{K}^{p_{K}-1}$ denote the matrix of the orthogonal projection from $\mathbb{R}_{p_{K}}(K)$ onto $\mathbb{R}_{p_{K}-1}(K)$; on triangular meshes, the action of this matrix on a vector consists in keeping the first $p_{K}\left(p_{K}+1\right) / 2$ components of the vector. Then, owing to (4.3), the function $\tilde{u}^{K}$, for each $K \in \mathcal{T}_{h}$, results from the components of the vector $\tilde{\mathbf{u}}_{K} \in \mathbb{R}^{p_{K}\left(p_{K}+1\right) / 2}$ such that

$$
\tilde{\mathbf{u}}_{K}=\left(\mathbb{A}_{K, K}^{p_{K}-1}\right)^{-1} \Pi_{K}^{p_{K}-1} \mathbb{A}_{K, K} \mathbf{u}_{K} \quad \forall K \in \mathcal{T}_{h},
$$

where $\mathbb{A}_{K, K}^{p_{K}-1}=\Pi_{K}^{p_{K}{ }^{-1}} \mathbb{A}_{K, K}\left(\Pi_{K}^{p_{K}-1}\right)^{t}$ is obtained simply from $\mathbb{A}_{K, K}$ by removing rows and columns corresponding to the basis functions of degree equal to $p_{K}$.

Finally, we define the error estimate for the projection $u_{h}^{\mathrm{p}-1}$ by

$$
\eta_{K}^{\mathrm{p}-1}=\left\|\nabla\left(u_{h}-u_{h}^{\mathrm{p}-1}\right)\right\|_{K} .
$$




\subsection{Two classical $h p$-decision criteria}

We recall two classical $h p$-decision criteria for which we use the same names as in the survey paper [31].

- PARAM: this criterion [23] hinges on the following local smoothness indicator:

$$
g_{K}:=\frac{\eta_{K}}{\eta_{K}^{\mathrm{p}-1}} \quad \forall K \in \mathcal{T}_{h}
$$

One expects that if the solution is not smooth in $K$, then $\left.u_{h}\right|_{K}$ is only slightly more accurate than $u_{h}^{\mathrm{p}-1}$ so that $g_{K} \approx 1$. On the other hand, if the solution is smooth in $K$, one expects that $g_{K} \ll 1$. Letting $\gamma \in(0,1)$, this leads to the following criterion: If $g_{K} \leq \gamma$, apply $p$-refinement, otherwise apply $h$-refinement. The presence of the user-defined parameter $\gamma$ is a drawback of this technique. In our numerical experiments, we use the two values $\gamma=0.3$ and $\gamma=0.6$.

- PRIOR: this criterion, which is closely inspired from [41], hinges on the smoothness indicator

$$
s_{K}:=1-\frac{\log \left(\eta_{K} / \eta_{K}^{\mathrm{p}-1}\right)}{\log \left(p_{K} /\left(p_{K}-1\right)\right)} .
$$

If $p_{K} \leq s_{K}-1$, we apply $p$-refinement, otherwise we apply $h$-refinement. The motivation is that one expects that the error indicators behave according to $\eta_{K} / \eta_{K}^{\mathrm{p}-1} \approx\left(p_{K} / p_{K}-1\right)^{-s_{K}-1}$, which follows from the a priori error bound $\left\|\nabla\left(u-u_{h}\right)\right\| \leq c h^{\min (p, s-1)} p^{1-s}\|u\|_{H^{s}(\Omega)}$ provided $u \in H^{s}(\Omega)$, cf. [4].

\subsection{A novel $h p$-decision criterion: DECAY}

Let $K \in \mathcal{T}_{h}$. Let $\eta_{h_{K}}^{p_{K}}$ denote the (estimate of) the error in $K$ where $h_{K}$ stands for the diameter of $K$ and $p_{K}$ for the polynomial degree in $K$. Considering triangular meshes, the number of degrees of freedom in $K$ is $\operatorname{DoF}_{h_{K}}^{p_{K}}=\frac{1}{2}\left(p_{K}+1\right)\left(p_{K}+2\right)$. Now, let us apply either $p$ - or $h$-refinement to $K$. The corresponding (estimates of the) errors are denoted by $\eta_{h_{K}}^{p_{K}+1}$ and $\eta_{h_{K} / 2}^{p_{K}}$, while the number of degrees of freedom becomes $\operatorname{DoF}_{h_{K}}^{p_{K}+1}=\frac{1}{2}\left(p_{K}+2\right)\left(p_{K}+3\right)$ and $\operatorname{DoF}_{h_{K} / 2}^{p_{K}}=2\left(p_{K}+1\right)\left(p_{K}+2\right)$, respectively. The idea is then to choose that refinement which leads to the steepest decrease of the error with respect to the number of degrees of freedom. Recalling (5.1), we evaluate the decay factors for $h$ - and $p$-refinements as follows:

$$
\beta_{\mathrm{p}}=\frac{\ln \left(\eta_{h_{K}}^{p_{K}} / \eta_{h_{K}}^{p_{K}+1}\right)}{\left(\mathrm{DoF}_{h_{K}}^{p_{K}+1}\right)^{\frac{1}{3}}-\left(\mathrm{DoF}_{h_{K}}^{p_{K}}\right)^{\frac{1}{3}}}, \quad \beta_{\mathrm{h}}=\frac{\ln \left(\eta_{h_{K}}^{p_{K}} / \eta_{h_{K} / 2}^{p_{K}}\right)}{\left(\mathrm{DoF}_{h_{K} / 2}^{p_{K}}\right)^{\frac{1}{3}}-\left(\mathrm{DoF}_{h_{K}}^{p_{K}}\right)^{\frac{1}{3}}} .
$$

Then, if $\beta_{\mathrm{p}}>\beta_{\mathrm{h}}$, we apply $p$-refinement, otherwise we apply $h$-refinement.

The difficulty lies in evaluating $\eta_{h_{K}}^{p_{K}+1}$ and $\eta_{h_{K} / 2}^{p_{K}}$. One possibility is to solve the problem on a globally $h p$-refined mesh, as in $[14,15,40]$, but this is quite timeconsuming as shown in [31]. Another possibility is the use of a higher-order reconstruction; see [18] for one example. Here we use a different idea in that the decay factors $\beta_{\mathrm{p}}$ and $\beta_{\mathrm{h}}$ are estimated using $p$ - and $h$-coarsening. The corresponding (estimates of the) errors are then denoted by $\eta_{h_{K}}^{p_{K}-1}$ and $\eta_{2 h_{K}}^{p_{K}}$, and the corresponding numbers of degrees 
of freedom become $\operatorname{DoF}_{h_{K}}^{p_{K}-1}=\frac{1}{2} p_{K}\left(p_{K}+1\right)$ and $\operatorname{DoF}_{2 h_{K}}^{p_{K}}=\frac{1}{8}\left(p_{K}+1\right)\left(p_{K}+2\right)$. Then, instead of (5.6), we use

$$
\beta_{\mathrm{p}}=\frac{\ln \left(\eta_{h_{K}}^{p_{K}} / \eta_{h_{K}}^{p_{K}-1}\right)}{\left(\mathrm{DoF}_{h_{K}}^{p_{K}-1}\right)^{\frac{1}{3}}-\left(\operatorname{DoF}_{h_{K}}^{p_{K}}\right)^{\frac{1}{3}}}, \quad \beta_{\mathrm{h}}=\frac{\ln \left(\eta_{h_{K}}^{p_{K}} / \eta_{2_{K}}^{p_{K}}\right)}{\left(\operatorname{DoF}_{2 h_{K}}^{p_{K}}\right)^{\frac{1}{3}}-\left(\mathrm{DoF}_{h_{K}}^{p_{K}}\right)^{\frac{1}{3}}} .
$$

In our numerical experiments, the quantity $\eta_{h_{K}}^{p_{K}-1}$ is evaluated as $\eta_{h_{K}}^{p_{K}-1}=\| \nabla\left(u_{h}-\right.$ $\left.u_{h}^{\mathrm{p}-1}\right) \|_{K}$ with the projection $u_{h}^{\mathrm{p}-1}$ devised in Section 5.1 , while the quantity $\eta_{2 h_{K}}^{p_{K}}$ is evaluated as $\eta_{2 h_{K}}^{p_{K}}=\left\|\nabla\left(u_{h}-\bar{u}^{K}\right)\right\|_{K}$ where $\bar{u}^{K}$ is the projection of $u_{h}$ in the $H^{1}(D(K))$-norm where $D(K)=\left\{K^{\prime} \in \mathcal{T}_{h} ; K^{\prime} \cap K \neq \emptyset\right\}$. Notice that the evaluation of $\bar{u}^{K}$ is less time-consuming than that of $\eta_{K}$.

\section{Numerical results}

In this section we assess the computational performance of our $h p$-adaptive algorithms on four benchmark problems from [30]. For the first three, we consider (2.1) with $\Gamma_{\mathrm{D}}=\partial \Omega$, while $f$ and $u_{D}$ are evaluated from the known exact solution. The fourth problem involves a piecewise constant, anisotropic diffusion tensor and has no known exact solution. We extend our estimates to this case following [10]. We illustrate the asymptotic exponential order of convergence (5.1) of the $h p$-decision criteria PARAM for $\gamma=0.3,0.6$, PRIOR, and DECAY. For the first two benchmark problems, we also include a comparison with the criterion IDEAL which exploits a priori knowledge of the singularities of the exact solution, namely $h$-refinement is used for all the marked elements which touch the (corner or line) singularities of the exact solution, while $p$-refinement is used for all the other marked elements. In all cases, we consider the incomplete IPDG method $(\vartheta=0)$ on triangular meshes, with the penalty parameter $\alpha=20$; notice that $\left\|\nabla u-\mathfrak{G}\left(u_{h}\right)\right\|=\left\|\nabla\left(u-u_{h}\right)\right\|$ in this case, cf. (3.26). The effectivity index of the a posteriori estimate is defined as $I^{\text {eff }}:=\frac{\eta}{\left\|\nabla\left(u-u_{h}\right)\right\|}$. Our adaptive algorithm produces sequences of (non-nested) triangular grids with hanging nodes, where the flux and potential reconstructions are evaluated on a global matching refinement of $\mathcal{T}_{h}$ as discussed in Remark 3.11.

\subsection{Case 1: re-entrant corner singularity}

Here $\Omega:=(-1,1)^{2} \backslash[0,1]^{2}, f=0$, and $u(r, \varphi)=r^{2 / 3} \sin (2 \varphi / 3)$, where $(r, \varphi)$ are the polar coordinates. Owing to the re-entrant corner, this problem features a singularity at the origin such that $u \in H^{5 / 3-\epsilon}(\Omega), \epsilon>0$. The presence of the singularity does not allow for faster convergence than $O\left(h^{2 / 3}\right)$ on uniformly refined grids. We carried out computations with $\varpi=10^{-4}$. The left and central panels of Figure 3 show the error decay with respect to $\mathrm{DoF}^{1 / 3}$ in logarithmic-linear scale for the various $h p$ decision criteria. For completeness, we include a comparison with pure $h$-refinement keeping $p_{K}=2$ fixed ( $h$-ADAPT). We observe that all $h p$-decision criteria lead to an asymptotic exponential convergence rate with minor differences. Moreover, the right panel of Figure 3 indicates that effectivity indices $I^{\mathrm{eff}}$ approach 1 (or a close value) with refinement. Figure 4 shows the final $h p$-grid obtained using DECAY with some details around the singularity. The final $h p$-grids obtained by all the methods are very similar for all the numerical examples. 

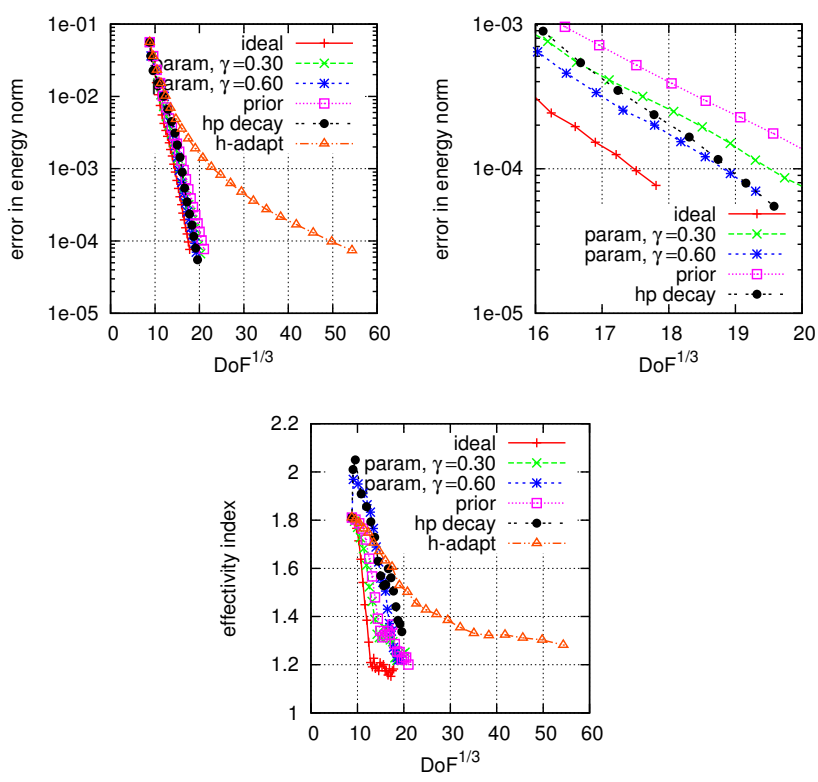

Fig. 3: Case 1, error decay with respect to $\mathrm{DoF}^{1 / 3}$ in logarithmic-linear scale for several $h p$-adaptive methods, global (left) and detailed (center) views and effectivity indices $I^{\text {eff }}$ (right). 


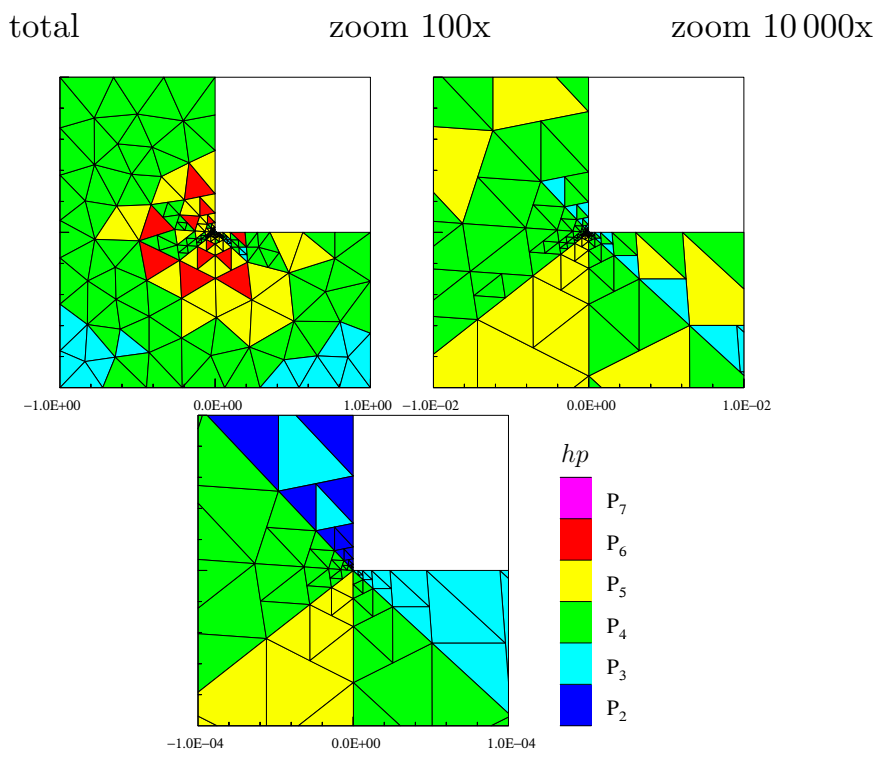

Fig. 4: Case 1, final $h p$-grids with details around the origin obtained using DECAY.

\subsection{Case 2: interior line singularity}

The setting is $\Omega=(-1,1)^{2}$ and

$$
u\left(x_{1}, x_{2}\right)= \begin{cases}\cos \left(\pi x_{2} / 2\right) & \text { for } x_{1} \leq \beta\left(x_{2}-1\right), \\ \cos \left(\pi x_{2} / 2\right)+\left(x_{1}-\beta\left(x_{2}-1\right)\right)^{\alpha} & \text { for } x_{1}>\beta\left(x_{2}-1\right),\end{cases}
$$

with $\alpha=2$ and $\beta=0.6$. The solution satisfies $u \in H^{5 / 2-\epsilon}(\Omega), \epsilon>0$, and features a mild singularity along the line $x_{1}-\beta\left(x_{2}-1\right)=0$. This example is more challenging than Case 1 since the line singularity is milder $\left(u \in H^{2}(\Omega)\right)$. The left panel of Figure 5 shows the isolines of the exact solution.

We carried out computations with $\varpi=10^{-4}$. Figure 6 shows the error decay with respect to $\mathrm{DoF}^{1 / 3}$ in logarithmic-linear scale for the various $h p$-decision criteria and the corresponding effectivity indices. We observe that all the $h p$-decision criteria lead to an asymptotic exponential convergence rate with small minor differences; the convergence of the method PRIOR is slightly slower. The effectivity indices are between 1 and 3 . Figure 7 shows the final $h p$-grid obtained using DECAY, with some details around the singularity.

\subsection{Case 3: multiple difficulties}

This test case combines a point singularity due to a re-entrant corner, a circular wave front (which might include a singularity at the center of the circle), a sharp peak, and 

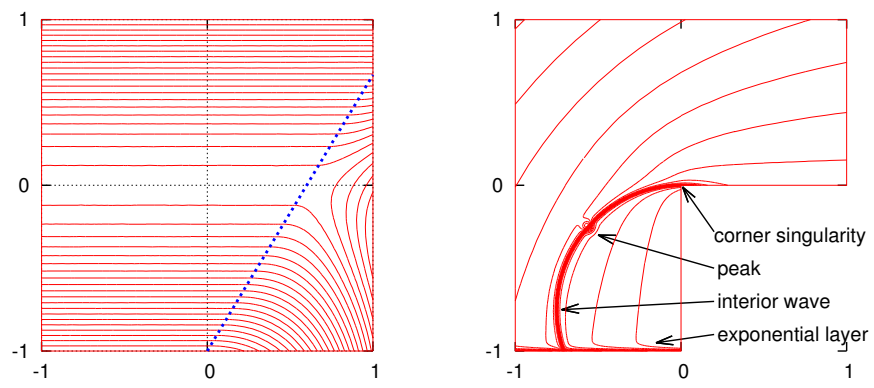

Fig. 5: Isolines of the exact solution for Case 2 (left) and Case 3 (right).
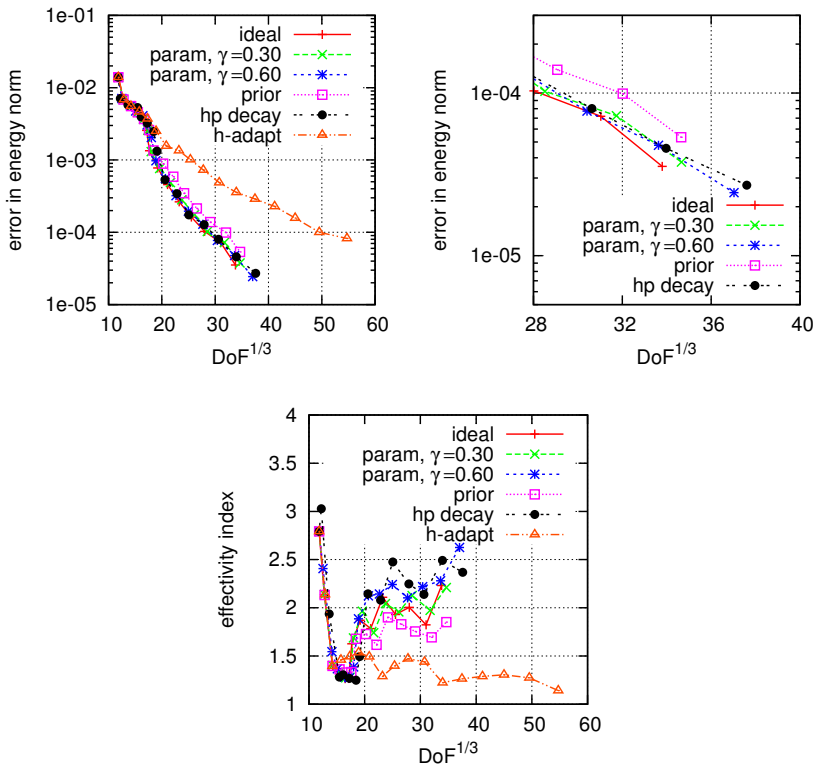

Fig. 6: Case 2, error decay with respect to $\mathrm{DoF}^{1 / 3}$ in logarithmic-linear scale for several $h p$-adaptive methods, global (left) and detailed (center) views and effectivity indices $I^{\text {eff }}$ (right). 

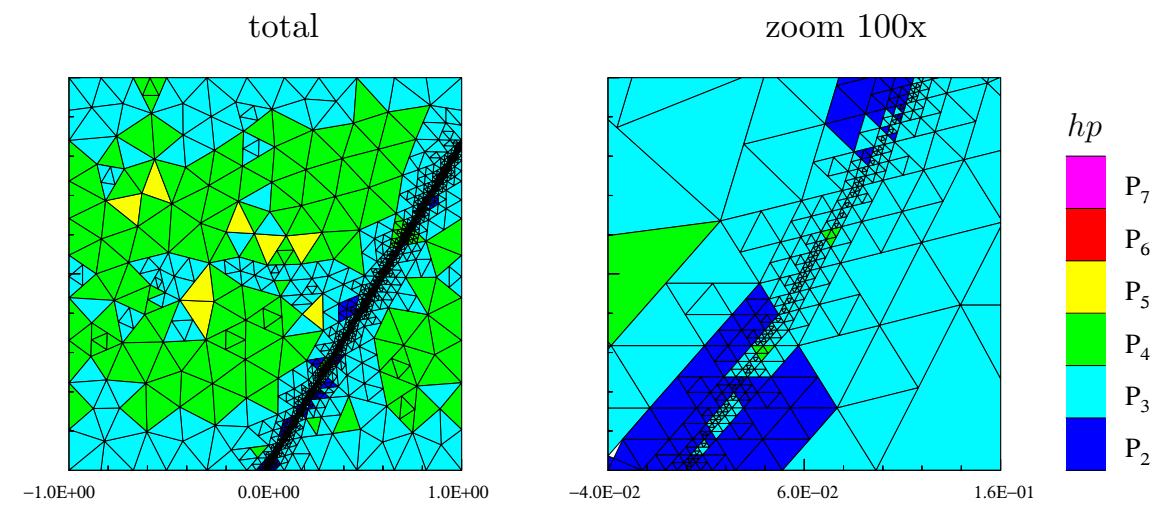

Fig. 7: Case 2, final $h p$-grids with details around the origin obtained with DECAY.

a boundary layer. The setting is $\Omega:=(-1,1)^{2} \backslash[0,1] \times[-1,1]$ and

$$
\begin{aligned}
u= & r^{\pi / \sigma} \sin (\varphi \pi / \sigma)+\tan ^{-1}\left\{\alpha_{w}\left[\left(\left(x_{1}-x_{2}^{w}\right)^{2}+\left(x_{2}-x_{2}^{w}\right)^{2}\right)^{1 / 2}-r_{w}\right]\right\} \\
& +\exp \left[-\alpha_{p}\left(\left(x_{1}-x_{2}^{p}\right)^{2}+\left(x_{2}-x_{2}^{p}\right)^{2}\right)\right]+\exp \left[-\alpha_{e}\left(1+x_{2}\right)\right]
\end{aligned}
$$

where the re-entrant angle is $\sigma=3 \pi / 2$. The interior wave is defined by $x_{1}^{w}=0$, $x_{2}^{w}=-3 / 4, r_{w}=3 / 4$, and $\alpha_{w}=200$. The peak is centered at $x_{1}^{p}=-\sqrt{5} / 4$, $x_{2}^{p}=-1 / 4$ with strength $\alpha_{p}=1000$. The boundary layer is given by $\alpha_{e}=100$. The right panel of Figure 5 shows the isolines of the exact solution together with a description of its main features. The salient difficulties are the steep interior wave and the exponential boundary layer, which behave like singularities on a coarse grid.

We carried out computations with $\varpi=10^{-1}$. Figure 8 shows the error decay with respect to $\mathrm{DoF}^{1 / 3}$ in logarithmic-linear scale for the various $h p$-decision criteria. We observe that all the $h p$-decision criteria lead to an asymptotic exponential convergence rate with small minor differences; here, DECAY and PARAM with $\gamma=0.6$ lead to somewhat faster convergence. The behavior of the effectivity indices indicates asymptotic exactness for all methods. Figure 9 shows the final $h p$-grid obtained using DECAY with some details around the singularity.

\subsection{Case 4: battery}

This example comes from [14]. We consider the elliptic problem $-\nabla \cdot(\mathbb{D} \nabla u)=f$ in $\Omega$, where the right-hand side $f$ and the diagonal diffusion matrix $\mathbb{D}=\operatorname{diag}\left(d_{1}, d_{2}\right)$ are piecewise constant on $\Omega$. The values of the triple $\left(f, d_{1}, d_{2}\right)$ for the colored subdomains from Figure 10 (left) are $(0,25,25)$ (red), (1,7,0.8) (green), (1,5,0.0001) (violet), $(0,0.2,0.2)$ (yellow), and $(0,0.05,0.05)$ (blue). On the left part of the boundary, we prescribe a homogeneous Neumann boundary condition and on the top, right, and bottom parts of the boundary, we prescribe the Dirichlet boundary condition $u_{\mathrm{D}}=1$. For the exact location of the line segments, we refer to [30, Section 2.5]. Since the right-hand side and the diffusion tensor are piecewise constant, the solution exhibits various singularities in the computational domain. The (approximate) solution of this problem is shown in the central panel of Figure 10. 

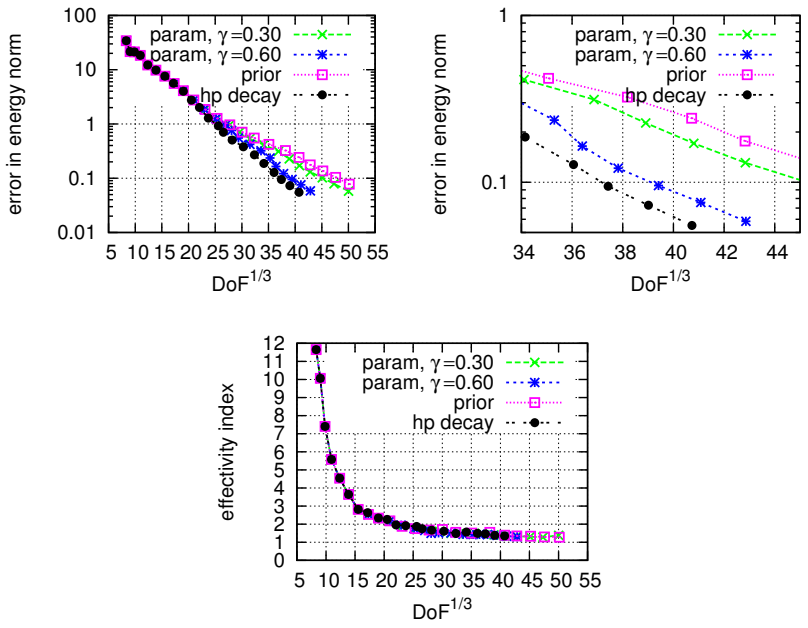

Fig. 8: Case 3, error decay with respect to $\mathrm{DoF}^{1 / 3}$ in logarithmic-linear scale for several $h p$-adaptive methods, global (left) and detailed (center) views and effectivity indices $I^{\text {eff }}$ (right).

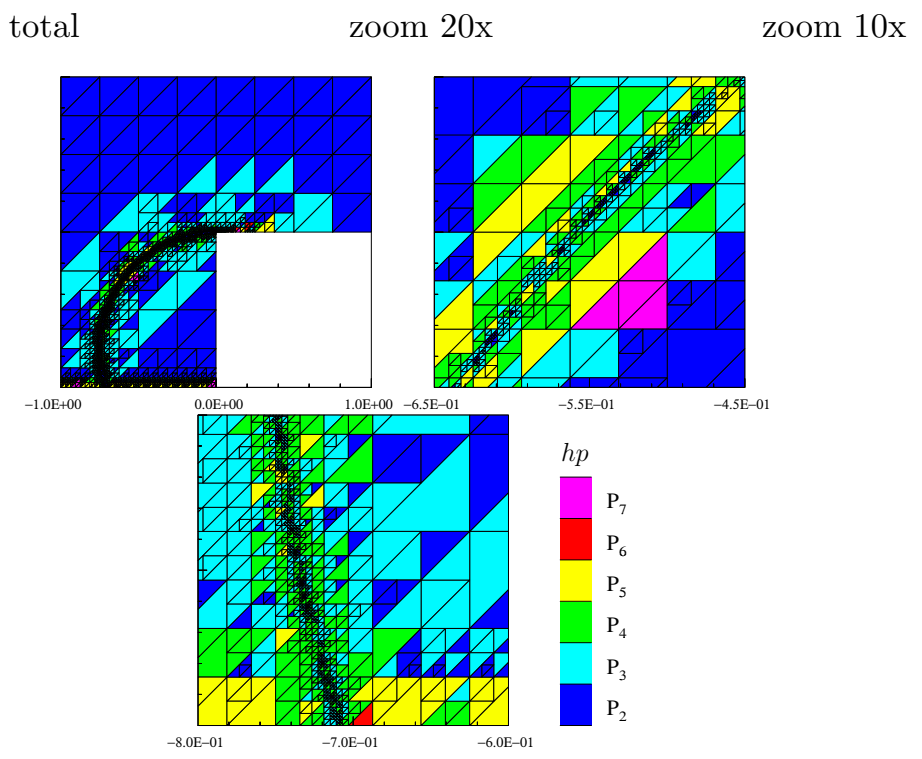

Fig. 9: Case 3, final $h p$-grids (left) with details around the peak (center) and the exponential boundary layer (right) obtained using DECAY. 

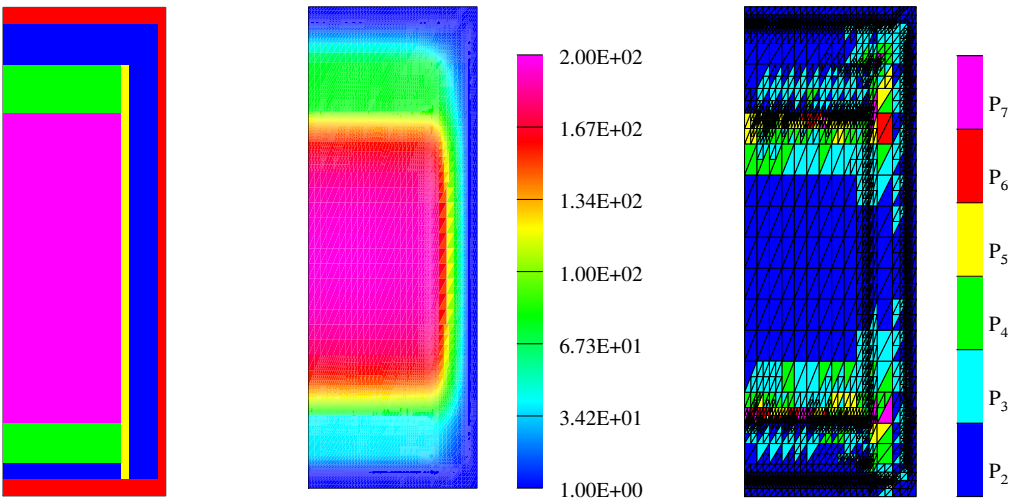

Fig. 10: Case 4, partition of the computational domain (left), final approximate solution (center), and final $h p$-grid obtained using DECAY (right).
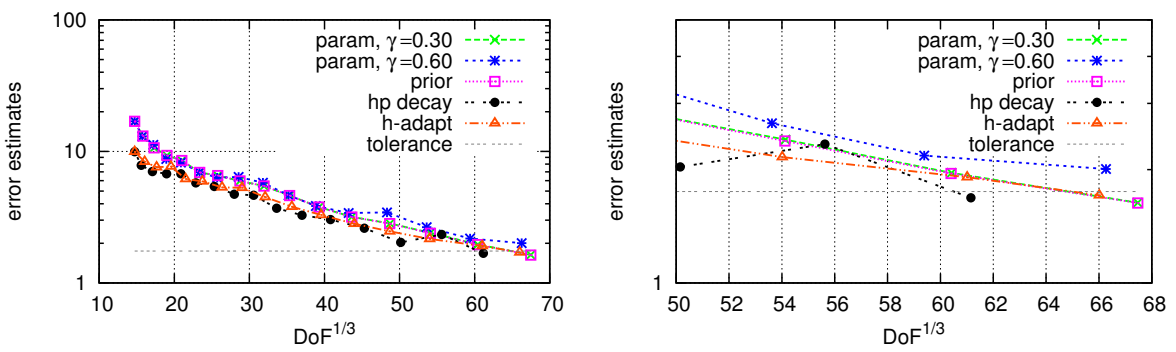

Fig. 11: Case 4, error decay with respect to $\mathrm{DoF}^{1 / 3}$ in logarithmic-linear scale for several $h p$-adaptive methods, global (left) and detailed (right) view.

We carried out computations with $\varpi=1.75$. Figure 11 shows the error decay with respect to $\mathrm{DoF}^{1 / 3}$ in logarithmic-linear scale for the various $h p$-decision criteria. All the criteria (including $h$-ADAPT) lead to an almost identical convergence behavior. This can be explained by the fact that interior singularities are dominant, so that $h$-refinement is essentially required everywhere. The right panel of Figure 10 shows the final $h p$-grid obtained using DECAY.

\subsection{Summary}

The selected cases indicate that all the tested $h p$-decision criteria lead to asymptotic exponential convergence rates. For the criterion PARAM, the disadvantage is the necessity to choose the parameter $\gamma$; a careful choice of $\gamma$ can produce superior convergence, but it is unclear how to find it. The criteria PRIOR and DECAY are parameter-free, and the second one turns out to perform slightly better. 


\section{References}

[1] S. Adjerid, M. Aiffa, AND J. E. Flaherty, Computational methods for singularly perturbed systems, in Analyzing multiscale phenomena using singular perturbation methods (Baltimore, MD, 1998), vol. 56 of Proc. Sympos. Appl. Math., Amer. Math. Soc., Providence, RI, 1999, pp. 47-83.

[2] M. Ainsworth And R. RAnkin, Fully computable error bounds for discontinuous Galerkin finite element approximations on meshes with an arbitrary number of levels of hanging nodes, SIAM J. Numer. Anal., 47 (2010), pp. 4112-4141.

[3] — Constant free error bounds for nonuniform order discontinuous Galerkin finite-element approximation on locally refined meshes with hanging nodes, IMA J. Numer. Anal., 31 (2011), pp. 254-280.

[4] I. BABUŠKA AND M. SURI, The hp-version of the finite element method with quasiuniform meshes, $\mathrm{M}^{2} \mathrm{AN}$ Math. Model. Numer. Anal., 21 (1987), pp. 199238.

[5] I. BABUŠKA AND M. SuRI, The $p$ and $h-p$ versions of the finite element method, basic principles and properties, SIAM Rev., 36 (1994), pp. 578-632.

[6] D. Braess, V. Pillwein, AND J. SchöBerl, Equilibrated residual error estimates are p-robust, Comput. Methods Appl. Mech. Engrg., 198 (2009), pp. 1189-1197.

[7] S. C. Brenner, Poincaré-Friedrichs inequalities for piecewise $H^{1}$ functions, SIAM J. Numer. Anal., 41 (2003), pp. 306-324.

[8] F. Brezzi And M. Fortin, Mixed and hybrid finite element methods, vol. 15 of Springer Series in Computational Mathematics, Springer-Verlag, New York, 1991.

[9] C. Carstensen And C. Merdon, Computational survey on a posteriori error estimators for nonconforming finite element methods for the Poisson problem, J. Comput. Appl. Math., 249 (2013), pp. 74-94.

[10] P. Ciarlet, JR. And M. Vohralík, Robust a posteriori error control for transmission problems with sign changing coefficients using localization of dual norms. HAL Preprint 01148476, submitted for publication, 2015.

[11] M. Costabel And A. McIntosh, On Bogovskiu and regularized Poincaré integral operators for de Rham complexes on Lipschitz domains, Math. Z., 265 (2010), pp. 297-320.

[12] L. Demkowicz, J. Gopalakrishnan, And J. SchöBerl, Polynomial extension operators. Part III, Math. Comp., 81 (2012), pp. 1289-1326.

[13] L. Demkowicz, W. Rachowicz, And P. Devloo, A fully automatic hpadaptivity, J. Sci. Comput., 17 (2002), pp. 117-142.

[14] L. F. Demkowicz, Computing with hp-adaptive finite elements. Vol. 1: Oneand two-dimensional elliptic and Maxwell problems., Applied Mathematics and Nonlinear Science Series, Chapman \& Hall/CRC, Boca Raton, FL, 2007.

[15] L. F. Demkowicz, J. Kurtz, D. Pardo, M. Paszyński, W. Rachowicz, AND A. ZDUnEK, Computing with hp-adaptive finite elements. Vol. II: Frontiers: Three-dimensional elliptic and Maxwell problems with applications., Applied Mathematics and Nonlinear Science Series, Chapman \& Hall/CRC, Boca Raton, FL, 2008.

[16] P. Destuynder And B. Métivet, Explicit error bounds in a conforming finite element method, Math. Comp., 68 (1999), pp. 1379-1396. 
[17] D. A. Di Pietro And A. ERn, Mathematical Aspects of Discontinuous Galerkin Methods, vol. 69 of Mathématiques \& Applications, Springer-Verlag, Berlin, 2011.

[18] V. Dolejší AND P. Šolín, hp-discontinuous Galerkin method based on local higher order reconstruction, Appl. Math. Comput., 279 (2016), pp. 219-235.

[19] T. Eibner AND J. M. MELENK, An adaptive strategy for hp-FEM based on testing for analyticity, Comput. Mech., 39 (2007), pp. 575-595.

[20] A. Ern, A. F. Stephansen, And M. Vohralík, Guaranteed and robust discontinuous Galerkin a posteriori error estimates for convection-diffusion-reaction problems, J. Comput. Appl. Math., 234 (2010), pp. 114-130.

[21] A. ERn And M. Vohralík, Polynomial-degree-robust a posteriori estimates in a unified setting for conforming, nonconforming, discontinuous Galerkin, and mixed discretizations, SIAM J. Numer. Anal., 53 (2015), pp. 1058-1081.

[22] T. Fankhauser, T. P. Wihler, And M. Wirz, The hp-adaptive FEM based on continuous Sobolev embeddings: isotropic refinements, Comput. Math. Appl., 67 (2014), pp. 854-868.

[23] W. Gui AND I. BABUŠKA, The $h, p$ and $h-p$ versions of the finite element method in 1 dimension. III. The adaptive h-p version, Numer. Math., 49 (1986), pp. 659683.

[24] P. Houston, D. Schötzau, And T. P. Wihler, Energy norm a posteriori error estimation of hp-adaptive discontinuous Galerkin methods for elliptic problems, Math. Models Methods Appl. Sci., 17 (2007), pp. 33-62.

[25] P. Houston And E. SüLI, A note on the design of hp-adaptive finite element methods for elliptic partial differential equations, Comput. Methods Appl. Mech. Engrg., 194 (2005), pp. 229-243.

[26] K.-Y. KIM, A posteriori error analysis for locally conservative mixed methods, Math. Comp., 76 (2007), pp. 43-66.

[27] P. LAdevèze And D. Leguillon, Error estimate procedure in the finite element method and applications, SIAM J. Numer. Anal., 20 (1983), pp. 485-509.

[28] J. M. MELENK, hp-interpolation of nonsmooth functions and an application to hp-a posteriori error estimation, SIAM J. Numer. Anal., 43 (2005), pp. 127-155.

[29] J. M. Melenk And B. I. Wohlmuth, On residual-based a posteriori error estimation in hp-FEM, Adv. Comput. Math., 15 (2001), pp. 311-331 (2002). A posteriori error estimation and adaptive computational methods.

[30] W. F. Mitchell, A collection of 2D elliptic problems for testing adaptive grid refinement algorithms, Appl. Math. Comput., 220 (2013), pp. 350-364.

[31] W. F. Mitchell And M. A. MCClain, A comparison of hp-adaptive strategies for elliptic partial differential equations, ACM Trans. Math. Software, 41 (2014), pp. Art. 2, 39.

[32] S. NicAise, A posteriori error estimations of some cell-centered finite volume methods, SIAM J. Numer. Anal., 43 (2005), pp. 1481-1503.

[33] G. V. Pencheva, M. Vohralík, M. F. Wheeler, and T. Wildey, Robust a posteriori error control and adaptivity for multiscale, multinumerics, and mortar coupling, SIAM J. Numer. Anal., 51 (2013), pp. 526-554.

[34] W. Prager and J. L. Synge, Approximations in elasticity based on the concept of function space, Quart. Appl. Math., 5 (1947), pp. 241-269. 
[35] S. I. REPIN, A posteriori estimates for partial differential equations, vol. 4 of Radon Series on Computational and Applied Mathematics, Walter de Gruyter GmbH \& Co. KG, Berlin, 2008.

[36] J. E. Roberts And J.-M. Thomas, Mixed and hybrid methods, in Handbook of Numerical Analysis, Vol. II, North-Holland, Amsterdam, 1991, pp. 523-639.

[37] C. Schwab, $p$ - and hp-Finite Element Methods, Clarendon Press, Oxford, 1998.

[38] P. Šlín, Partial differential equations and the finite element method, Pure and Applied Mathematics, Wiley-Interscience, New York, 2004.

[39] P. Šolín And L. Demkowicz, Goal-oriented hp-adaptivity for elliptic problems, Comput. Methods Appl. Mech. Engrg., 193 (2004), pp. 449-468.

[40] P. Šolín, K. Segeth, And I. DoležEl, Higher-order finite element methods, Studies in Advanced Mathematics, Chapman \& Hall/CRC, Boca Raton, FL, 2004.

[41] E. Süli, P. Houston, And C. Schwab, hp-finite element methods for hyperbolic problems, in The mathematics of finite elements and applications, X, MAFELAP 1999 (Uxbridge), Elsevier, Oxford, 2000, pp. 143-162.

[42] A. Veeser And R. Verfürth, Poincaré constants for finite element stars, IMA J. Numer. Anal., 32 (2012), pp. 30-47.

[43] R. VERFÜRTH, A posteriori error estimation techniques for finite element methods, Numerical Mathematics and Scientific Computation, Oxford University Press, Oxford, 2013.

[44] M. VoHRALÍK, Unified primal formulation-based a priori and a posteriori error analysis of mixed finite element methods, Math. Comp., 79 (2010), pp. 2001-2032. 